\title{
Concepts in CMB Anisotropy Formation
}

\author{
Wayne $\mathrm{Hu}$ \\ Institute for Advanced Study, Princeton, NJ 08540
}

"To divide is to leave something undivided. To discriminate between alternatives is to leave something which is neither alternative."

Chuang-tzu

\begin{abstract}
:
These lecture notes form a primer on the theory of cosmic microwave background (CMB) anisotropy formation. With emphasis on conceptual aspects rather than technical issues, we examine the physical foundations of anisotropy evolution in relativistic kinetic and perturbation theory as well as the manifestation of these principles in primary and secondary anisotropies. We discuss gauge choice and gauge invariance and their use in understanding the CMB. Acoustic, gravitational redshift and ionization effects have robust signatures in the CMB spectrum and may allow determination of classical cosmological parameters as well as reveal general distinctions between models for structure formation. We develop the tight and weak coupling approximations as analytic tools to help understand these effects and the robustness of their signatures.
\end{abstract}

whu@sns.ias.edu

IASSNS-AST 95/52

To appear in The Universe at High-z, Large Scale Structure and the Cosmic Microwave Background, eds. E. Martinez-Gonzalez and J.L Sanz (Springer Verlag, in press) 


\section{Introduction}

More than three years has passed since cosmic microwave background (CMB) anisotropies were first detected at large angular scales in the COBE DMR sky maps (Smoot et al. 1992). Since then much progress has been made in probing the anisotropy spectrum at smaller scales (see e.g. the compilations by Bond 1995; Scott, Silk \& White 1995; Steinhardt 1995). Satellite missions now being proposed would be able to map the anisotropy definitively down to a fraction of a degree. To exploit these recent and potential experimental advances, we need an accurate and accessible theory of anisotropy formation. The first part of this task was largely accomplished in the 1980's with the ground-breaking work of Wilson \& Silk (1981), Vittorio \& Silk (1984), and Bond \& Efstathiou (1984). Detailed numerical calculations in perturbation theory allow accurate predictions of the anisotropy in most models for structure formation. Summaries of the current state of the art in numerical codes can be found in Bond (1995), Hu et al. (1995) and Ma \& Bertschinger (1995).

Numerical work however is notoriously inaccessible to the uninitiated. Moreover, it obscures the true potential of CMB anisotropy spectrum for cosmology by limiting itself to specific models. Indeed, CMB anisotropies are sensitive to classical cosmology parameters such as the matter and radiation content, baryon fraction, expansion rate, curvature, and cosmological constant (Bond et al. 1994; Seljak 1994; Hu \& Sugiyama 1995a) as well as the model for structure formation ( $\mathrm{Hu} \&$ Sugiyama 1995b; Albrecht et al. 1995, Crittenden \& Turok 1995; Durrer et al. 1995; Magueijo et al. 1995). In these lecture notes, we will review the basic concepts of anisotropy formation and their consequence for cosmology.

We begin in $\S 2$ with relativistic kinetic and perturbation theory which give the basic physical laws that govern anisotropy formation. Pure reductionism of this sort however would miss the true use of CMB anisotropies. In $\S 3$ and $\S 4$, we map out the manifestations of the basic principles before and after recombination, i.e. the primary and secondary anisotropies. These anisotropies possess information on both the cosmological model and structure formation. From this decomposition and reconstruction, the resulting complex structure of anisotropies can be understood in a general model-independent manner. 


\section{Physical Foundation}

Two ingredients are necessary to describe the evolution of cosmic microwave background anisotropies: relativistic kinetic and perturbation theory. Kinetic theory describes the radiation transport properties of the CMB photons in the metric perturbed by gravitationally unstable density fluctuations. These fluctuations are evolved through relativistic perturbation theory. Combined, the two considerations yield a complete system that is responsible for anisotropy formation in any model where structure formation proceeds by gravitational instability.

\subsection{Relativistic Kinetic Theory}

Conceptually, relativistic kinetic theory is identical to the familiar nonrelativistic case: the phase space distribution is conserved along geodesics save for a collision term due to scattering,

$$
\frac{d f}{d t} \equiv \frac{\partial f}{\partial t}+\frac{\partial f}{\partial x^{i}} \frac{d x^{i}}{d t}+\frac{\partial f}{\partial p} \frac{d p}{d t}+\frac{\partial f}{\partial \gamma^{i}} \frac{d \gamma^{i}}{d t}=C[f]
$$

Here $\gamma_{i}$ are the direction cosines of the photon momentum $p$. Before a redshift of $z_{*} \approx 1000, \mathrm{CMB}$ photons were hot enough to ionize hydrogen. Consequently, the dominant interaction process for the CMB at early times was Compton scattering off free electrons. Due to the higher electron density in the early universe, the Compton mean free path was quite short, much smaller than the particle horizon at that time. The horizon at $z_{*}$ subtends a degree or less for $\Omega_{0}+\Omega_{\Lambda} \lesssim 1$. Thus on scales relevant for observable anisotropies, the photons were tightly coupled to the electrons, which in turn were tightly coupled to the protons by Coulomb interactions. At $z_{*}$, neutral hydrogen formed through "recombination" and the photons last scattered. Unless the universe suffered reionization at high redshift, fluctuations in the $\mathrm{CMB}$ at recombination were frozen in at $z_{*}$ and await observation today.

There are several features of Compton scattering worth noting

1. Scattering couples the photons to the baryons and forces perturbations in their number and hence energy density to evolve together.

2. Scattering isotropizes the photons in the electron rest frame thus coupling the local CMB dipole to the electron velocity.

3. In the Thomson limit, there is no energy transfer in scattering. Energy exchange only occurs to $\mathcal{O}\left(\left\langle v_{e}^{2}\right\rangle\right)$, i.e. $\mathcal{O}\left(T_{e} / m_{e}\right)$.

4. There is no change in photon number through Compton scattering.

Scattering thus governs the intrinsic temperature perturbations at last scattering and the dipole or bulk velocity perturbation. Since it does not change the net energy or photon number in the CMB to lowest order, spectral distortions to the blackbody do not arise in linear theory. Unless the electrons 
have been heated significantly above the temperature of the CMB or photons and/or energy has been dumped into the CMB from an external source, the spectral information can be ignored.

The remaining subtlety is that the photons propagate in a space-time that is distorted by density fluctuations: we must employ the geodesic equation in the presence of perturbations. This leads to gravitational redshift effects from the $d p / d t$ term in equation (1) which can also generate fluctuations in the CMB. The $d \gamma^{i} / d t$ term represents gravitational lensing and gives a first order contribution only in a curved universe (see §3.4). By integrating equation (1) over frequencies, one obtains the Boltzmann equation for the evolution of temperature perturbations $\Theta(\eta, \mathbf{x}, \gamma) \equiv \Delta T / T$,

$$
\left[\frac{\partial}{\partial \eta}+\dot{x}^{i} \frac{\partial}{\partial x^{i}}+\dot{\gamma}^{i} \frac{\partial}{\partial \gamma^{i}}\right] \Theta=S_{G}+S_{C}
$$

where $S_{G}$ and $S_{C}$ are the sources from the gravitational redshift and Compton scattering and overdots are derivatives with respect to conformal time $\eta=\int d t / a$. For a derivation from first principles of the Compton collision term $S_{C}$, see Hu, Scott \& Silk (1994), Dodelson \& Jubas (1995), and Kosowsky (1995). If the angular dependence and polarization of Compton scattering is ignored it reduces to $\dot{\tau}\left[\Theta_{0}-\Theta-\gamma_{i} v_{e}^{i}\right]$. Here $\Theta_{0}$ is the isotropic temperature fluctuation and the differential Compton optical depth $\dot{\tau}=x_{e} n_{e} \sigma_{T} a$ with $x_{e}$ as the ionization fraction, $n_{e}$ as the electron density and $\sigma_{T}$ as the Thomson cross section. Implicit in $S_{G}$ and $S_{C}$ are the three fundamental sources of anisotropies in the CMB:

1. Gravitational redshifts from the presence and evolution of metric fluctuations.

2. Hot and cold spots from the intrinsic temperature at last scattering.

3. The Doppler effect due to the velocity of the last scatterers.

Of course, this high level description is not very practical. Gravitational instability controls the evolution of density, velocity, and metric perturbations in the universe. All of these effects are thus related by relativistic perturbation theory.

\subsection{Relativistic Perturbation Theory}

General relativity tells us that matter moves in a space-time perturbed by fluctuations in the matter density itself. Thus the Einstein equations reduce conceptually into two pieces. The stress-energy tensor of the total matter is covariantly conserved in the perturbed metric, $T^{\mu \nu} ; \mu=0$, and matter fluctuations are the source of metric perturbations via a generalized Poisson equation. For the former, $\nu=0$ gives number or energy density conservation, i.e. the continuity equation. The spatial components give momentum conservation, i.e. the Euler equation. 


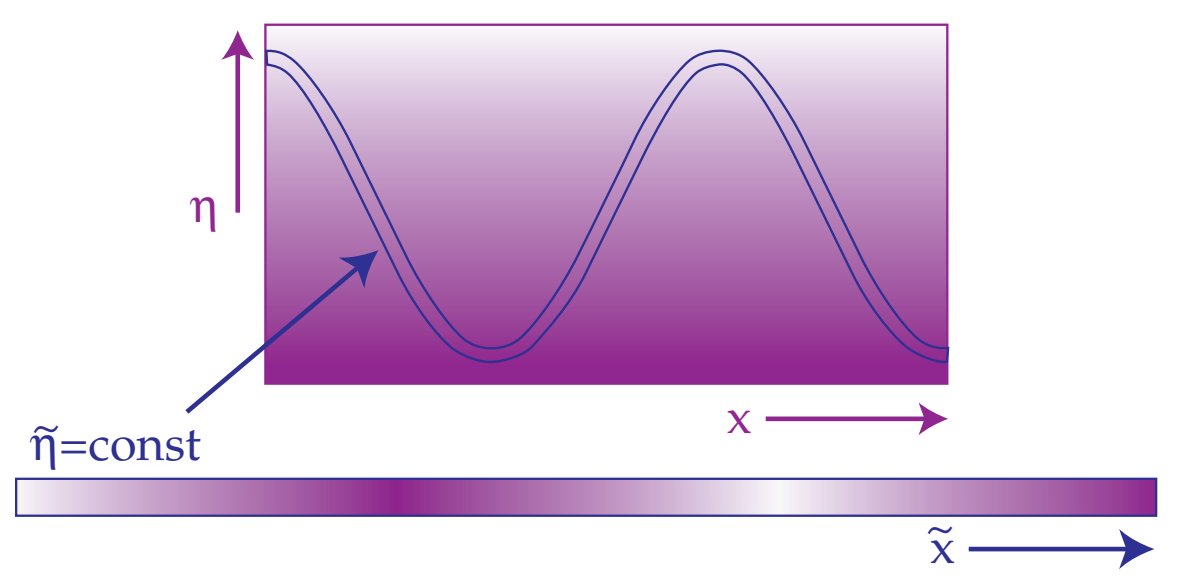

Fig. 1. Gauge ambiguity. Due to the change in density with the expansion, even a homogeneous FRW universe can appear to have a density perturbation if the time slicing is warped. The synchronous and total matter gauges choose the time slicing to correspond to the rest frame of the freely falling and total matter respectively. The Newtonian gauge chooses slicing such that the expansion rate is shear free.

\subsubsection{Gauge Choice}

To explicitly implement these principles, we need to treat a subtlety due to gauge freedom in general relativistic perturbation theory. In order to define a perturbation, we must specify the relation between the physical spacetime and the hypothetical unperturbed background. The difference between quantities at the same coordinate values is deemed a perturbation. Since this choice is not unique, one must generally fix a gauge before making any calculations or interpretations regarding perturbation evolution.

Practically speaking, a gauge choice involves fixing the constant-time hypersurfaces and the spatial grid on these surfaces. Suppose we warp the time slicing from some fiducial choice by $\tilde{\eta}=\eta+T e^{i \mathbf{k} \cdot \mathbf{x}}$ (see Fig. 1). The density of a fluid of particle $X$ on this slicing becomes $\tilde{\rho}_{X}=\rho_{X}-\dot{\rho}_{X} T e^{i \mathbf{k} \cdot \mathbf{x}}$ for the same coordinate value. Because the expansion makes densities decrease in time as

$$
\dot{\rho}_{X}=-3\left(\rho_{X}+p_{X}\right) \frac{\dot{a}}{a},
$$

in the new frame the density fluctuation is

$$
\tilde{\delta}_{X}=\delta_{X}+3\left(1+p_{X} / \rho_{X}\right) \frac{\dot{a}}{a} T e^{i \mathbf{k} \cdot \mathbf{x}} .
$$

As a simple example of gauge ambiguity, consider a Friedmann-RobertsonWalker universe with no perturbations $\delta_{X}=0$. Even in this case, a density fluctuation appears in the warped time slicing. One might argue that this involves a silly choice of time slicing and that observers may set up a sensible coordinate system that removes ambiguities by exchanging light signals. 
This is true. All "sensible" choices of coordinate systems agree on the nature and value of the density perturbation within the horizon by requiring that the warping satisfy $\delta \eta / \eta=T / \eta \ll \delta$ if $k \eta \gg 1$. However outside the horizon, there is no "sensible" way to set up the coordinate system. In particular, different choices of time slicing can produce seemingly different results for perturbations and their evolution in this regime.

There are two basic approaches to fixing a gauge or coordinate system. We can choose a set of preferred observers and set the coordinate frame to be their rest frame, i.e. employ "Lagrangian" coordinates. The popular and useful synchronous gauge is one such representation. Here we choose freely falling observers to define the frame, e.g. the collisionless cold dark matter. This rest frame analysis is convenient in that it simplifies the evolution equations and their solution. An extension of this idea is employed in the total matter gauge. Here the coordinate system coincides with the rest frame of the combined relativistic and non-relativistic matter. This choice is computationally useful in the early universe where radiation dominates and does not necessarily follow the perturbations in the non-relativistic matter.

The computational convenience of these "Lagrangian" techniques is offset by difficulties in obtaining physical intuition for processes such as anisotropy formation. Perturbations grow by dilation effects due to distortions of the coordinate grid rather than simple causal mechanisms such as gravitational infall and redshift. An alternate choice is to take an "Eulerian" coordinate system. More specifically, instead of employing a set of preferred observers, fix the coordinate system by a geometric property. One useful choice is the Newtonian gauge. The coordinate frame here corresponds to zero-shear hypersurfaces where the expansion appears isotropic. Inside the horizon, this analysis reduces to the standard non-relativistic Newtonian treatment. Thus, our physical intuition from the non-relativistic theory most easily carries over to a complete treatment in this gauge.

Since there is a one-to-one mapping between gauges whose coordinates are entirely fixed, these treatments are entirely equivalent and give the same result for any observable quantity, e.g. CMB anisotropies. Ambiguities only arise if the coordinate system is not entirely fixed. In this case, the oneto-many mapping produces unphysical gauge modes which historically has produced much confusion (see Press \& Vishniac 1980). Gauge modes can be avoided by completely fixing the coordinates. The definitions of the total matter and Newtonian gauges already do so. On the other hand, a synchronous coordinate system, defined by a set of freely falling observers, requires us to specify which set through the initial conditions. In particular, if the initial velocity of the observers is fixed then there are no gauge modes. In practice, one usually takes the rest frame of the CDM. 


\subsubsection{Gauge Invariance}

Due to the computational convenience of the "Lagrangian" gauges and the intuitive nature of the "Eulerian" ones, it is often useful to switch between gauges in the midst of a calculation or even employ different gauge representations in a single set of evolution equations. A useful technique for this purpose is provided by the gauge invariant method (Bardeen 1980; Kodama \& Sasaki 1984; Mukhanov, Feldman \& Brandenberger 1992). It is called gauge invariant not because a density perturbation, say, remains a density perturbation under a gauge transformation. Rather, a density perturbation in a specific gauge is represented in a coordinate free manner, i.e. in a manner valid in an arbitrary gauge. In practical terms, this just means that whatever gauge is chosen, one has a systematic way to mix in quantities that represent perturbations in a different gauge.

Let us make these considerations more concrete. The most general form of a metric perturbed by scalar fluctuations a given Fourier mode $k$ is (Bardeen 1980)

$$
\begin{aligned}
g_{00} & =-a^{2}\left[1+2 A^{G} e^{i \mathbf{k} \cdot \mathbf{x}}\right], \\
g_{0 j} & =a^{2} B^{G} i \hat{k}_{j} e^{i \mathbf{k} \cdot \mathbf{x}}, \\
g_{i j} & =a^{2}\left\{\delta_{i j}+\left[2 H_{L}^{G} \delta_{i j}+2 H_{T}^{G}\left(-\hat{k}_{i} \hat{k}_{j}+\delta_{i j} / 3\right)\right] e^{i \mathbf{k} \cdot \mathbf{x}}\right\},
\end{aligned}
$$

where for illustration purposes, we have taken a flat geometry and superscript $G$ is meant to remind the reader that these quantities are gauge dependent. By fixing the gauge, we can eliminate two out of the four metric terms. In the Newtonian gauge, $B^{N}=H_{T}^{N}=0$, and it is traditional to label the Newtonian potential $A^{N}=\Psi$ and the space curvature perturbation $H_{L}^{N}=\Phi$. Synchronous gauge requires $A^{S}=B^{S}=0$, whereas the total matter gauge takes $B^{T}=V_{T}^{T}$ and $H_{T}^{T}=0$ where $V_{T}^{T}$ is the velocity of the total matter. Ordinarily, one now writes down the Einstein equations $G_{\mu \nu}=8 \pi G T_{\mu \nu}$ which contain the conservation $T_{; \mu}^{\mu \nu}=0$ and "Poisson" equations, with a specific choice of gauge. For a fluid of particle $X$, the stress energy momentum tensor is

$$
\begin{aligned}
& T_{0}^{0}=-\left(1+\delta_{X}^{G} e^{i \mathbf{k} \cdot \mathbf{x}}\right), \\
& T^{0}{ }_{i}=\left(\rho_{X}+p_{X}\right) V_{X}^{G}\left(-i \hat{k}_{i}\right) e^{i \mathbf{k} \cdot \mathbf{x}}, \\
& T^{i}{ }_{j}=\left(p_{X}+\delta p_{X}^{G} e^{i \mathbf{k} \cdot \mathbf{x}}\right) \delta^{i}{ }_{j}+p_{X} \Pi_{X}\left(-\hat{k}^{i} \hat{k}_{j}+\delta^{i}{ }_{j}\right) e^{i \mathbf{k} \cdot \mathbf{x}},
\end{aligned}
$$

where $\Pi_{X}$ is the anisotropic stress of the fluid.

As an example of the gauge invariant program, let us write the total matter gauge density perturbation in a gauge-independent way. The total matter gauge condition is satisfied by shifting the time slicing from an arbitrary gauge by $T=\left(V_{T}^{G}-B^{G}\right) / k$ [Kodama \& Sasaki 1984, eq. (3.3a); $\mathrm{Hu}$ 1995, eq. (4.88)]. Equation (3) tells us that the density perturbation transforms as 


$$
\delta_{X}^{T}=\delta_{X}^{G}+3\left(1+p_{X} / \rho_{X}\right) \frac{\dot{a}}{a}\left(V_{T}^{G}-B^{G}\right) / k .
$$

In an arbitrary gauge $G$, this represents what the density perturbation would be in the total matter rest frame. This "gauge invariant" definition can be used to employ total matter gauge variables in a Newtonian or synchronous gauge treatment:

$$
\begin{aligned}
\delta_{X}^{T} & =\delta_{X}^{N}+3 \frac{\dot{a}}{a}\left(1+p_{X} / \rho_{X}\right) V_{T}^{N} / k \\
& =\delta_{X}^{S}+3 \frac{\dot{a}}{a}\left(1+p_{X} / \rho_{X}\right) V_{T}^{S} / k .
\end{aligned}
$$

If fluctuations are adiabatic, the number density fluctuations of the matter and radiation evolve together, i.e. their bulk velocities are equal. In this case, $V_{T}^{S}$ is equal to the freely-falling cold dark matter velocity, commonly defined to be zero in synchronous gauge. Equation (7) then tells us that the total matter gauge and synchronous gauge density perturbation are numerically equivalent. In a matter dominated (pressureless) universe, there is no fundamental scale in these rest frame evolution equations and density fluctuations evolve in a scale free manner. This is not true for the Newtonian gauge. Here fluctuations grow by the gravitational infall of the matter into potential wells. Due to causality, this introduces a fundamental scale, the horizon scale, into the evolution equations. For example in the case of adiabatic fluctuations, density perturbations are constant outside the horizon and only grow (or decay) after the horizon has grown larger than the wavelength.

Now let us see how gauge modes creep in. The mapping of synchronous perturbations onto any gauge with fixed coordinates is unambiguous as we have seen. However, since it is a many-to-one operation, its inverse leaves additional gauge freedom. Notice from equation (7), knowledge of the total matter or Newtonian gauge perturbations only fixes a certain combination of density and velocity perturbations in synchronous gauge. To remove the ambiguity, one must fix the initial synchronous velocity. Since an initial velocity decays with the expansion as $a^{-1}$, equation (7) tells us that a different choice will alter the behavior of densities by an additional term proportional to $\dot{a} / a^{2}$ or $a^{-2}$ during radiation domination and $a^{-3 / 2}$ during matter domination. This is a gauge mode.

In summary, the "gauge invariant" approach does nothing to solve the gauge ambiguity; no solution is necessary since gauge choice poses no fundamental problems. Yet even though there is nothing particularly deep about the "gauge invariant" program, it is often useful. It allows us to borrow Newtonian and rest frame concepts for use in any gauge. In addition, by providing a systematic way of mapping perturbations in a specific gauge onto an arbitrary gauge, one automatically determines whether the coordinates have been completely fixed, i.e. whether gauge modes have been entirely eliminated. 


\subsection{Newtonian Gauge Equations}

Now we are ready to state the evolution equations in an explicit form. To avoid unnecessary confusion, we will stay in the pure Newtonian gauge throughout this treatment. We will hereafter drop the superscript $N$ with the understanding that all perturbation variables are in the Newtonian gauge unless otherwise specified. The continuity and Euler equations for the photon temperature in flat space are

$$
\begin{aligned}
& \dot{\Theta}_{0}=-\frac{k}{3} \Theta_{1}-\dot{\Phi} \\
& \dot{\Theta}_{1}=k\left[\Theta_{0}+\Psi-\frac{1}{6} \Pi_{\gamma}\right]-\dot{\tau}\left(\Theta_{1}-V_{b}\right),
\end{aligned}
$$

where the term proportional to the Compton differential optical depth $\dot{\tau}$ comes from momentum conservation in the scattering. Recall that $\Theta_{0}=\frac{1}{4} \delta_{\gamma}$ is the isotropic temperature fluctuation, and $\Theta_{1}=V_{\gamma}$ is the amplitude of the photon dipole or bulk velocity. One can see that when the optical depth to scattering is high, the photons become isotropic in the electronbaryon rest frame, i.e. the dipole moment $\Theta_{1}=V_{b}$. The anisotropic stress of the photons $\Pi_{\gamma}$ is directly proportional to its quadrupole moment. Since scattering makes the photons isotropic in the baryon rest frame, the photon anisotropic stress is negligible before recombination. These equations can also be directly obtained from kinetic theory. In fact, they are contained in the Legendre decomposition of the Boltzmann equation (2) in Fourier space which also gives the evolution of $\Pi_{\gamma}$ through a hierarchy of higher angular moments (Wilson \& Silk 1981).

Aside from the usual velocity divergence source in the continuity equation, there is a term dependent on the metric. This is due to the gravitational redshift effects of time dilation. As the form of the metric $g_{i j}=$ $-a^{2} \delta_{i j}\left(1+2 \Phi e^{i \mathbf{k} \cdot \mathbf{x}}\right)$ implies, it is entirely analogous to the cosmological redshift. Heuristically, the presence of matter curves or stretches space taking the wavelength of the photon with it. In the Euler equation, the Newtonian potential $\Psi$ acts as a source of the dipole. Gradients in the potential also induce gravitational blue and red shifts as the photons fall into and climb out of potential wells $(\Psi<0)$. This is countered by photon pressure from $\Theta_{0}$. As the temperature rises so does the pressure which opposes the fall of a photon into the potential well. As the photons free stream, power in the dipole is converted into the higher multipole moments through the quadrupole via the $\Pi_{\gamma}$ term.

The baryon continuity and Euler equations take on a similar form

$$
\begin{aligned}
\dot{\delta}_{b} & =-k V_{b}-3 \dot{\Phi} \\
\dot{V}_{b} & =-\frac{\dot{a}}{a} V_{b}+k \Psi+\dot{\tau}\left(\Theta_{1}-V_{b}\right) / R .
\end{aligned}
$$


Momentum conservation in Compton scattering gives the form of the coupling. From equation (5), the effective momentum density of a general fluid $X$ is $\left(\rho_{X}+p_{X}\right) V_{X}$. Since $p_{\gamma}=\frac{1}{3} \rho_{\gamma}$ and $p_{b} \ll \rho_{b}$, conservation implies $\left(4 \rho_{\gamma} / 3\right) \delta \Theta_{1}=\rho_{b} \delta V_{b}$. Thus the Compton coupling for the baryons takes on a similar form to the protons but is of opposite sign and is altered by a factor of $R=3 \rho_{b} / 4 \rho_{\gamma}$.

Again, the continuity equation is modified by gravitational effects from the stretching of space associated with $\Phi$. Since the density decreases as the length scale cubed, the total differential effect becomes $3 \dot{\Phi}$. In the Euler equation, the velocity is damped by the expansion and enhanced by infall into potential wells. Particle momenta scale as $a^{-1}$ due to the expansion. For non-relativistic particles, this causes the peculiar velocity to scale similarly in the absence of sources. In the fully-relativistic case, it causes the temperature to decrease as $a^{-1}$.

Decoupled components such as the massless neutrinos and cold dark matter follow identical evolution equations save for the absence of Compton coupling. The metric perturbations on the other hand feel the influence of the total matter perturbations, $\rho_{T} \delta_{T}=\sum_{i} \rho_{i} \delta_{i},\left(\rho_{T}+p_{T}\right) V_{T}=\sum_{i}\left(\rho_{i}+\right.$ $\left.p_{i}\right) V_{i}$, and $p_{T} \Pi_{T}=\sum_{i} p_{i} \Pi_{i}$, where $i$ runs through all the particle species, through the generalized Poisson equations,

$$
\begin{aligned}
k^{2} \Phi & =4 \pi G a^{2} \rho_{T}\left[\delta_{T}+3 \frac{\dot{a}}{a}\left(1+p_{T} / \rho_{T}\right) V_{T} / k\right], \\
k^{2}(\Psi+\Phi) & =-8 \pi G a^{2} p_{T} \Pi_{T},
\end{aligned}
$$

which arise from the time-time + time-space and traceless space-space components of the Einstein equations respectively. The presence of $a^{2}$ in the first equation represents the conversion from physical to comoving coordinates. Notice that if we rewrite the equation in terms of the density fluctuation on the total matter rest frame, the first equation would take on the familiar non-relativistic form of the Poisson equation and simplify perturbation calculations. The additional term represents a relativistic effect that is important outside the horizon. When anisotropic stress $p_{T} \Pi_{T}$ may be ignored, the second of equations (10) reduces to $\Psi=-\Phi$ as one would expect for the Newtonian potential.

\subsection{Gauge Tricks: Sachs-Wolfe Example}

Examining the photon conservation equations (8) and the Poisson equations (10), we see that the former are simpler in the Newtonian gauge, whereas the latter are simpler in the total matter gauge. Let us see how gauge tricks developed

in $\S 2.2$ can help us understand their joint evolution. In the matter dominated epoch, the Poisson and continuity equations reduce to 


$$
\Phi=\frac{6}{(k \eta)^{2}} \delta_{T}^{T}, \quad \dot{\delta}_{T}^{T}=-k V_{T}
$$

as one expects from a non-relativistic analysis. Since $\delta_{T}^{T}$ is the density perturbation on the matter rest frame, its evolution in the growing mode also satisfies the non-relativistic relation $\delta_{T}^{T} \propto \eta^{2}$. Hence $V_{T}=-2 \delta_{T}^{T} / k \eta$. Recalling its relation to the Newtonian density perturbation, we obtain

$$
\begin{aligned}
\delta_{T} & =\delta_{T}^{T}-\frac{6}{k \eta} V_{T}=\left[1+\frac{12}{(k \eta)^{2}}\right] \delta_{T}^{T} \\
& \approx \frac{12}{(k \eta)^{2}} \delta_{T}^{T}=2 \Phi, \quad k \eta \ll 1 .
\end{aligned}
$$

For adiabatic fluctuations, the intrinsic temperature fluctuation, $\Theta_{0} \equiv$ $\frac{1}{4} \delta_{\gamma}=\frac{1}{3} \delta_{T}=\frac{2}{3} \Phi$. We shall see that after a gravitational redshift of $\Psi$, this implies that the effective temperature $\Theta_{0}+\Psi=\frac{2}{3} \Phi+\Psi=\frac{1}{3} \Psi$ which is the famous Sachs-Wolfe (1968) result.

\section{Primary Anisotropies and the Tight Coupling Approximation}

Before recombination, the Compton scattering time was so short that the photons and baryons behaved as a single fluid. This allows us to greatly simplify the treatment of anisotropy formation. Specifically, since the mean free path is much shorter than a wavelength of the fluctuation, the optical depth through a wavelength $\sim \dot{\tau} / k$ is large. Thus the evolution equations may be expanded in $k / \dot{\tau}$. Employing the baryon Euler equation (9), we may eliminate the baryon velocity from the photon evolution equation to obtain the first order equation (Peebles \& Yu 1970; Hu \& Sugiyama 1995a)

$$
\frac{d}{d \eta}(1+R) \dot{\Theta}_{0}+\frac{k^{2}}{3} \Theta_{0}=-\frac{k^{2}}{3}(1+R) \Psi-\frac{d}{d \eta}(1+R) \dot{\Phi} .
$$

where we have dropped the higher order correction $\Pi_{\gamma}=\mathcal{O}(k / \dot{\tau})$ (see $\S 3.2$ ). Conceptually, this equation reads: the change in momentum of the photonbaryon fluid is determined by a competition between the pressure restoring and the gravitational driving forces. If we ignore the time dependence of the baryon-photon momentum ratio $R$ from the expansion, this equation describes a forced harmonic oscillator. Since scattering requires the bulk velocities of the photons and baryons to be equal, the effective dimensionless mass of the fluid is given by $m_{\mathrm{eff}}=1+R$ to account for the inertia of the baryons. Baryons also contribute gravitational mass to the system as is

evident in the appearance of $m_{\text {eff }}$ in the infall and dilation terms on the right hand side. They do not however contribute significantly to the pressure or restoring force of the system. 


\subsection{Acoustic Oscillations}

As an instructive first approximation, let us ignore time variations in the potentials $\Phi$ and $\Psi$ and also the baryon-photon momentum ratio $R$ compared with changes at the oscillation frequency $\omega=k c_{s}$, where the sound speed (Doroshkevich, Zel'dovich \& Sunyaev 1978)

$$
c_{s}=\frac{1}{\sqrt{3(1+R)}} .
$$

Equation (11) then reduces to the familiar form

$$
\ddot{\Theta}_{0}+k^{2} c_{s}^{2} \Theta_{0}=-\frac{1}{3} k^{2} \Psi
$$

This is a simple harmonic oscillator under the constant acceleration provided by gravitational infall and can immediately be solved as

$$
\Theta_{0}(\eta)=\left[\Theta_{0}(0)+(1+R) \Psi\right] \cos \left(k r_{s}\right)+\frac{1}{k c_{s}} \dot{\Theta}_{0}(0) \sin \left(k r_{s}\right)-(1+R) \Psi
$$

where the sound horizon $r_{s}=\int c_{s} d \eta \approx c_{s} \eta$. The two initial conditions $\Theta_{0}(0)$ and $\dot{\Theta}_{0}(0)$ govern the form of the acoustic oscillation. We shall see below that they represent the adiabatic and isocurvature modes respectively. Equation (12) also implies through the photon continuity equation (8) that

$$
\Theta_{1}(\eta)=3\left[\Theta_{0}(0)+(1+R) \Psi\right] c_{s} \sin \left(k r_{s}\right)+3 k^{-1} \dot{\Theta}_{0}(0) \cos \left(k r_{s}\right) .
$$

In equations (12) and (13), lie the main acoustic and redshift effects which dominate primary anisotropy formation.

\subsubsection{Gravitational Infall and Redshift}

In the early universe, photons dominate the fluid and $R \rightarrow 0$. In this limit, the oscillation takes on an even simpler form. For the adiabatic mode, $\dot{\Theta}_{0}(0)=0$ and $\Theta_{0}(\eta)=\left[\Theta_{0}(0)+\Psi\right] \cos \left(k r_{s}\right)-\Psi$. This represents an oscillator with a zero point which has been displaced by gravity. The zero point represents the state at which gravity and pressure are balanced. The displacement $-\Psi>0$ yields hotter photons in the potential well since gravitational infall not only increases the number density of photons but also their energy through gravitational blueshifts.

However, photons also suffer a gravitational redshift from climbing out of the potential well after last scattering. This precisely cancels the $-\Psi$ blueshift. Thus, the effective temperature perturbation is $\Theta_{0}(\eta)+\Psi=$ $\left[\Theta_{0}(0)+\Psi\right] \cos \left(k r_{s}\right)$ (see Fig. 2). The phase of the oscillation at last scattering determines the effective fluctuation. Since the oscillation frequency $\omega=k c_{s}$, the critical wavenumber $k=\pi / r_{s}\left(\eta_{*}\right) \approx \pi / c_{s} \eta_{*}$ is essentially at the scale of the sound horizon. If there is a spectrum of $k$-modes, there will 

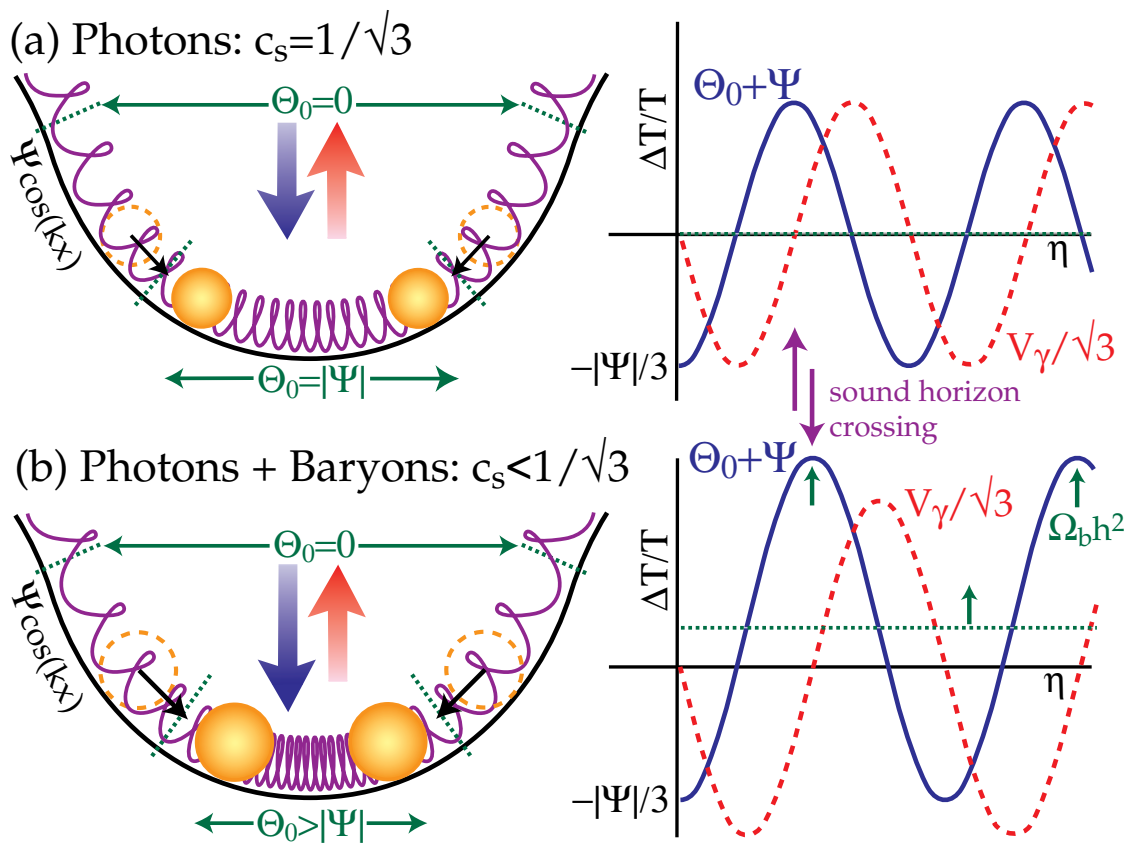

Fig. 2. Acoustic oscillations. Fluid compression through gravitational infall is resisted by photon pressure setting up acoustic oscillations. Without baryons the photon blueshift on infall is equal and opposite to the redshift upon leaving the potential leading to symmetric oscillations around zero with a Doppler effect of equal magnitude but 90 degrees out of phase. Baryons increase the mass of the fluid causing more infall and a net zero point displacement. Temperature crests (compression) are enhanced over troughs (rarefaction) and velocity contributions.

be a harmonic series of temperature fluctuation peaks with $k_{m}=m \pi / r_{s}\left(\eta_{*}\right)$ for the $m$ th peak. Odd peaks thus represent the compression phase (temperature crests), whereas even peaks represent the rarefaction phase (temperature troughs), inside the potential wells.

As first calculated by Sachs \& Wolfe (1967), the effective temperature in the matter dominated limit goes to $\Theta_{0}+\Psi=\frac{1}{3} \Psi$. In the Newtonian frame, this is a direct consequence of the adiabatic initial conditions. In general, if $k \eta \ll 1$ equation (8) implies

$$
\left[\Theta_{0}+\Psi\right]\left(\eta_{*}\right)=\left[\Theta_{0}+\Psi\right](0)+\left.[\Psi-\Phi]\right|_{0} ^{\eta_{*}} .
$$

In a full calculation, the small variation in the potential at equality due to the change in the equation of state brings the effective temperature from $\frac{1}{2} \Psi$ to $\frac{1}{3} \Psi$. As this does not affect the qualitative picture, we will encorporate this effect as $\left[\Theta_{0}+\Psi\right](0)=\frac{1}{3} \Psi$ and $\Delta(\Psi-\Phi)=0$. Notice that if $\Theta_{0}(0)=$ $\Psi(0)=-\Phi(0)=0$, then $\left[\Theta_{0}+\Psi\right]\left(\eta_{*}\right)=\Psi\left(\eta_{*}\right)-\Phi\left(\eta_{*}\right) \approx 2 \Psi\left(\eta_{*}\right)$ which yields a factor of 6 enhancement for isocurvature models. An alternate way of 


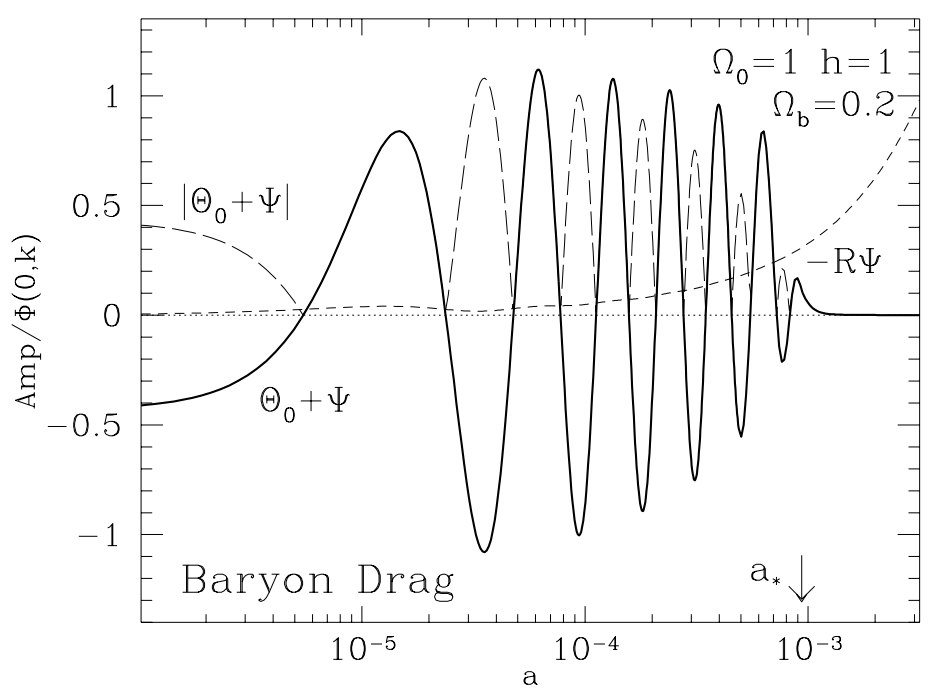

Fig. 3. Baryon Drag. Baryons drag the photons into potential wells leading to a zero point displacement of $|R \Psi|$ and alternating peak heights in the rms fluctuation which provide a measure of $\Omega_{b} h^{2}$. The fall off near $a_{*}$ of the amplitude is due to diffusion damping.

deriving these results is to employ gauge tricks as shown in $\S 2.4$. The SachsWolfe effect is a combination of an intrinsic temperature and a gravitational redshift. Since the photon density and thus the intrinsic temperature is a gauge dependent concept, this breakdown, but not the observable result, will also depend on gauge.

\subsubsection{Baryon Drag}

Though effectively pressureless, the baryons still contribute to the inertial and gravitational mass of the fluid $m_{\mathrm{eff}}=1+R$. This decreases the sound speed and changes the balance of pressure and gravity. Gravitational infall now leads to greater compression of the fluid in a potential well, i.e. a further displacement of the oscillation zero point (see Fig. 2). Since the redshift is not affected by the baryon content, this relative shift remains after last scattering to enhance all peaks from compression over those from rarefaction. If the baryon-photon ratio $R$ were constant, the effective temperature perturbation would become

$$
\Theta(\eta)+\Psi=\frac{1}{3} \Psi(1+3 R) \cos \left(k r_{s}\right)-R \Psi
$$

with compressional peaks a factor of $(1+6 R)$ over the $R=0$ case. In reality, the effect is reduced since $R \rightarrow 0$ at early times (see Fig. 3).

The evolution of the effective mass has another effect on its own. In classical mechanics, the ratio of energy $\frac{1}{2} m_{\mathrm{eff}} \omega^{2} A^{2}$ to frequency $\omega$ of an 


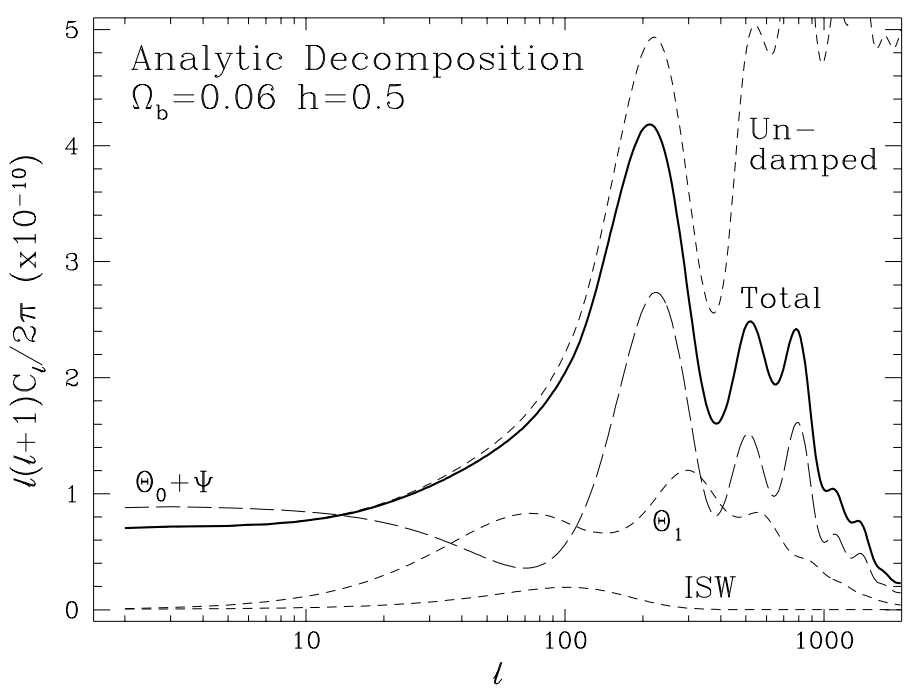

Fig. 4. Analytic decomposition $\left(\Omega_{0}=1\right.$ scale invariant adiabatic model $)$. The effective temperature after gravitational redshift dominates the primary anisotropy. Peak heights are enhanced and modulated by baryon-photon ratio $R$ as well as experience a boost crossing the equality scale at $\ell \approx \sqrt{3}\left(2 \Omega_{0} H_{0}^{2} / a_{e q}\right)^{1 / 2} \eta_{0} \approx 400$. The Doppler effect is smaller and out of phase with the temperature. The ISW effect due to potential decay after recombination is small here but can be significant for low matter content universes. Diffusion damping cuts off the acoustic spectrum at small scales.

oscillator is an adiabatic invariant. Thus for the slow changes in $m_{\mathrm{eff}} \propto c_{s}^{-2}$, the amplitude of the oscillation varies as $A \propto c_{s}^{1 / 2} \propto(1+R)^{-1 / 4}$ since $\omega \propto c_{s}$. The fundamental solutions of the oscillator equation are modified to be $(1+R)^{-1 / 4} \cos \left(k r_{s}\right)$ and $(1+R)^{-1 / 4} \sin \left(k r_{s}\right)$.

\subsubsection{Doppler Effect}

Since the turning points are at the extrema, the fluid velocity oscillates 90 degrees out of phase with the density (see Fig. 2). Its motion relative to the observer causes a Doppler shift. Whereas the observer velocity creates a pure dipole anisotropy on the sky, the fluid velocity causes a spatial temperature variation $\Theta_{1} / \sqrt{3}$ on the last scattering surface from its line of sight component. For a photon-dominated $c_{s} \approx 1 / \sqrt{3}$ fluid, equation (13) tells us the velocity contribution is equal in amplitude to the density effect. This photon-intrinsic Doppler shift should be distinguished from the electron-induced Doppler shift of reionized scenarios.

The addition of baryons significantly changes the relative velocity contribution. As the effective mass increases, conservation of energy requires that the velocity decreases for the same initial temperature displacement. Thus the relative amplitude of the velocity scales as $c_{s}$. In the toy model of 
a constant baryon-photon density ratio $R$, the oscillation becomes $\Theta_{1} / \sqrt{3}=$ $\frac{1}{3} \Psi(1+3 R)(1+R)^{-1 / 2} \sin \left(k r_{s}\right)$. Notice that velocity oscillations are symmetric around zero unlike the temperature ones. Thus compressional peaks will rise clearly above the velocity oscillations if $R$ is large. Even in a universe with $\Omega_{b} h^{2}$ given by nucleosynthesis, $R$ is sufficiently large to make velocity contributions subdominant (see Fig. 4).

\subsubsection{Acoustic Driving Effects and Isocurvature Models}

Whenever the non-relativistic matter is not the dominant dynamical component, the potentials $\Phi$ and $\Psi$ become time-dependent. For example, when the universe is radiation dominated, pressure and entropy alter the behavior of the gravitational potential. External sources from topological defects can also play a role. The effects of potential evolution can be separated into those that occur before last scattering and thus affect the acoustic oscillations and those that occur afterwards which affect the gravitational redshift of the photons along their free-streaming trajectories. We will defer consideration of the latter to $\S 4$ where we consider effects between recombination and the present.

As a simple example of the driving effects of potential evolution, let us again consider adiabatic fluctuations where the initial temperature $\Theta_{0}(0)$ and curvature fluctuations $\Phi(0)$ are finite constants related by the Poisson equation and $\dot{\Theta}_{0}(0)=0$. Inside the sound horizon, pressure prevents gravitational infall in the photon-baryon and neutrino systems. Unless CDM dominates, energy density perturbations $\delta \rho_{T}$ decay with the expansion and consequently can no longer maintain a constant gravitational potential. Counterintuitively, this decaying potential can actually enhance temperature fluctuations through its near resonant driving force. Since the potential decays after sound horizon crossing, it drives the first compression without a counterbalancing effect on the subsequent rarefaction stage (see Fig. 5).

Furthermore, the dilation effect from a decaying space curvature $\Phi$ also enhances the acoustic fluctuations. Heuristically, the overdensities which establish the potential well "stretch" the space-time fabric. As the potential well decays, it re-contracts. Photons which are caught in this contraction find their wavelength similarly contracted, i.e. blueshifted. Thus a differential change in $\Phi$ leads to a dilation effect, $\dot{\Theta}_{0}=-\dot{\Phi}$. Combined, the driving effects of infall and dilation yield an effect of order $\frac{1}{3} \Psi-\Psi+\Phi \approx \frac{5}{3} \Psi$ or 5 times the Sachs-Wolfe effect when the perturbation crosses the sound horizon (see Fig. 4,5 and $\mathrm{Hu} \&$ Sugiyama 1995c). Since this effect only occurs for modes which cross before CDM domination, the amplitude of this boost is sensitive to the matter-radiation ratio of the universe. In particular, lowering $\Omega_{0} h^{2}$, raising the massless neutrino number or energy density, or making some of the cold dark matter relativistic at early times through eV mass neutrinos (see Fig. 6b; Ma \& Bertschinger 1995; Dodelson, Gates \& 


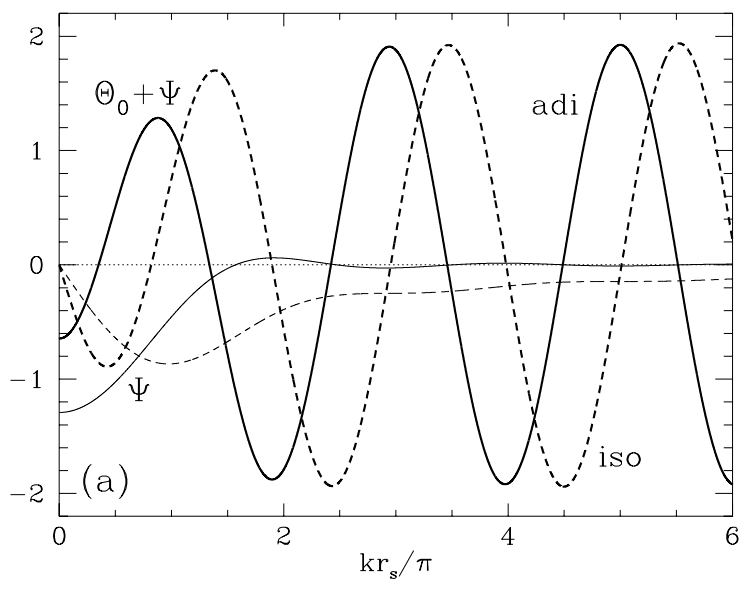

(b) Driving Effect

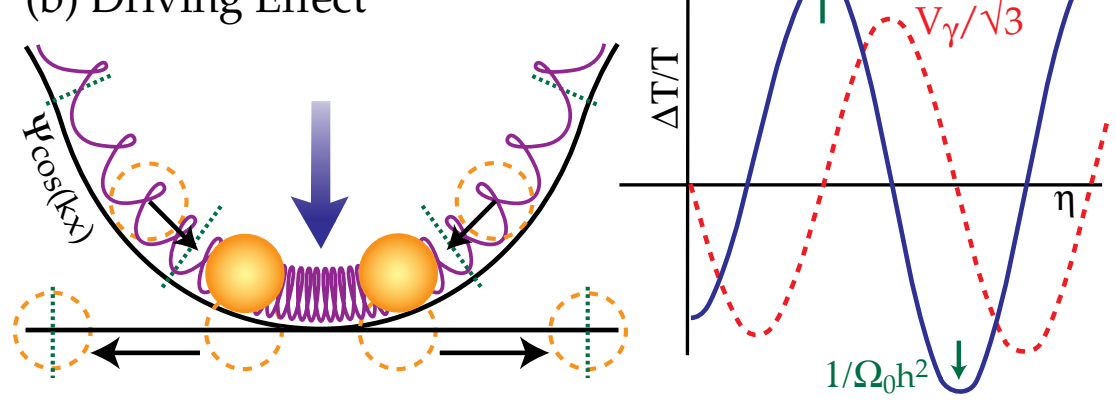

Fig. 5. Driving effects. The time evolution of the potential can enhance the amplitude of the acoustic oscillation by driving effects. In the adiabatic case [(a) solid lines, numerical results and (b) heuristic picture], the potential enhances the first compression through infall and decays leaving the oscillator strongly displaced from the zero point. In the (baryon) isocurvature case [(a) dashed line], the potential grows from zero and stimulates a sine mode. The first extrema here is suppressed due to the fact that the gravitation driving begins near sound horizon crossing. Notice that for the first cycle of the adiabatic and isocurvature oscillations, the gravitational force mimics a driving force of approximately twice the natural period.

Stebbins 1995), can move the boost to larger scales and thus enhance the first few acoustic peaks.

Similar resonant effects can occur in other situations. For example, many alternate scenarios follow a typical isocurvature behavior. The hallmark of isocurvature type models is that curvature fluctuations $\Phi$ are zero or at least suppressed outside the horizon. The curvature fluctuation grows by causal processes as the fluctuation crosses the horizon. This isocurvature behavior occurs for entropy fluctuations where matter and radiation perturbations are counterbalanced in the initial state, whether through the baryons $(\mathrm{Hu}$ \& Sugiyama 1995b), hot dark matter (de Laix \& Scherrer 1995) or axions 

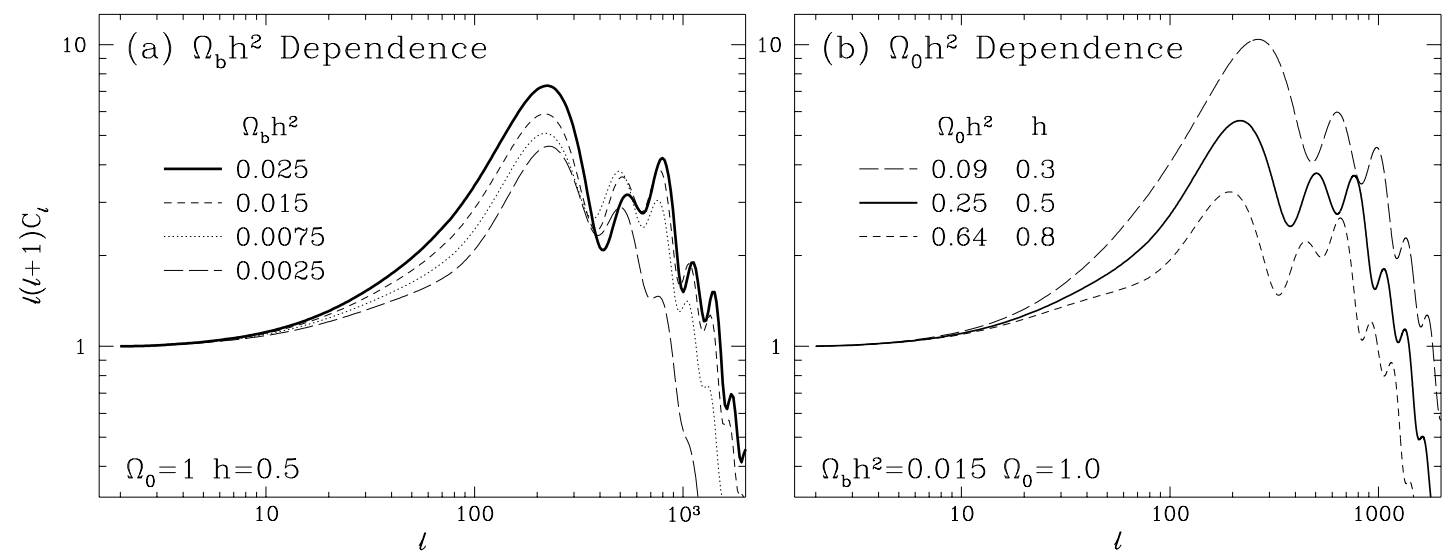

Fig. 6. Heights of the peaks in a scale invariant adiabatic model. The heights of the peaks is determined by the baryon drag and acoustic driving effects and so are sensitive to $\Omega_{b} h^{2}$ and $\Omega_{0} h^{2}$. Baryons increase compression in potential wells causing the peak heights to alternate. Lower matter content causes more potential decay driving the oscillation.

(Sugiyama \& Gouda 1992), texture models (Crittenden \& Turok 1995, Durrer, Gangui \& Sakellariadou 1995), and is at least part of the story for string models (Hindmarsh, private communication, but see also Albrecht, Coulson $\&$ Ferreira 1995). The qualitative effect on isocurvature conditions is easy to see. Since the potential grows from zero to a maximum near sound horizon crossing and then decays due to radiation pressure, the force drives the sine harmonic of oscillations (see Fig. 5a). The result is that isocurvature acoustic peaks are 90 degrees out of phase with their adiabatic counterparts. The first peak occurs at larger scales that the corresponding adiabatic peak but generally has a suppressed amplitude since the corresponding mode is just at the sound horizon $k r_{s}=\pi / 2$ and has not experienced the full effect of the driving term. These features may serve to distinguish the two basic types of scenarios in a manner independent of the details of the given model.

Notice that in these examples, oscillations give a coherent spectrum of peaks in integral or half integral multiples of $\int \omega d \eta=k r_{s}\left(\eta_{*}\right)$. Since each $k$ mode evolves independently, this coherence in the temporal phase is the result of a special timing in the gravitational impulse. In all the examples considered above, the gravitational force kicks in between horizon crossing and sound horizon crossing. This explains the coherence in $k$ and the fundamental scale in the problem $r_{s}\left(\eta_{*}\right)$. More exotic scenarios may not preserve this coherence. If the source of the gravitational potential has a more random temporal behavior, the sine and cosine acoustic modes will be stimulated incoherently with equal likelihood. Combined, the two modes would lead to a smoothed out rms temperature fluctuation below the sound horizon. Preliminary calculations indicate that this may be case for cosmic 
string models due to complicated behavior of the string network inside the horizon (Albrecht et al. 1995; Magueijo et al. 1995).

These considerations can be made quantitative by solving the oscillator equation under the influence of an arbitrary but known gravitational forcing function either analytically ( $\mathrm{Hu} \&$ Sugiyama 1995a) or numerically by modeling the gravitational source (e.g. Seljak 1994; Crittenden \& Turok 1995). Simple closed form solutions for standard adiabatic and isocurvature models are presented in $\mathrm{Hu} \&$ Sugiyama (1995c).

\subsection{Diffusion Damping}

In reality, the photons and baryons are not perfectly coupled since the photons possess a mean free path in the baryons $\lambda_{C} \approx \dot{\tau}^{-1}$ due to Compton scattering. As the photons random walk through the baryons, hot spots and cold spots are mixed. Fluctuations thereafter remain only in the unscattered fraction causing a near exponential decrease in amplitude as the diffusion length $\lambda_{D} \sim \sqrt{N} \lambda_{C}=\sqrt{\eta \lambda_{C}}$ or $k_{D} \sim \sqrt{\dot{\tau} / \eta}$ overtakes the wavelength.

To be more specific, diffusion causes heat conduction and generates viscosity in the fluid (Weinberg 1972). As photons from regions of different temperature meet, anisotropies form leading to a quadrupole moment or anisotropic stress in the fluid. If the diffusion length is well under the horizon, it overtakes the wavelength of the fluctuation when $\dot{\tau} / k=k \eta \gg 1$. Thus the optical depth through a wavelength is still high and the perturbative expansion of $\S 3.1$ still holds. This fact allows us to extend the tight coupling approximation for acoustic modes to last scattering. For small scale modes in which the optical depth through a wavelength $\dot{\tau} / k$ is not high at recombination, all acoustic oscillations will already have damped away $(\mathrm{Hu}$ \& Sugiyama 1995c).

Photon diffusion is a second order effect and damps acoustic oscillations as $\exp \left[-\left(k / k_{D}\right)^{2}\right]$ with the damping wavenumber

$$
k_{D}^{-2}=\frac{1}{6} \int d \eta \frac{1}{\dot{\tau}} \frac{R^{2}+4 f_{2}^{-1}(1+R) / 5}{(1+R)^{2}},
$$

where $f_{2}$ accounts for the subtle effects in the generation of anisotropic stress (see Fig. 4). Kaiser (1983) showed that $f_{2}=9 / 10$ due to the angular dependence and $3 / 4$ if the additional effects of polarization are included. The two processes aid the generation of the quadrupole moment and hence increase the viscous damping of the acoustic oscillations.

The baryons are dynamically coupled to the photons by momentum exchange in Compton scattering from $\dot{\tau}_{d} \equiv \dot{\tau} / R$ in equation (9). If $\dot{\tau}_{d} / k \gg 1$, the baryons will be dragged in and out of potential wells with the photons. This process destroys the baryonic acoustic oscillations as well and is known as Silk (1968) damping. Since coupling requires coevolution in the number 
density $\dot{\delta}_{b}=\frac{3}{4} \dot{\delta}_{\gamma}=3 \dot{\Theta}_{0}$, only entropy fluctuations $S=\delta_{b}-\frac{3}{4} \delta_{\gamma}$ survive diffusion damping.

Notice also that the ionization history factors in the diffusion length through the mean free path $\dot{\tau}^{-1}$. As we shall now see, at recombination the mean free path and hence the diffusion length increases substantially but does not approach the horizon scale.

\subsection{Decoupling}

The CMB anisotropy today is simply the acoustic fluctuation at last scattering, modified by diffusion damping and free streamed to the present:

$$
[\Theta+\Psi]\left(\eta_{0}, k, \mu\right) \approx\left[\Theta_{0}+\Psi-i \mu \Theta_{1}\right]\left(\eta_{*}, k\right) \mathcal{D}_{\gamma}(k) e^{i k \mu\left(\eta_{*}-\eta_{0}\right)}
$$

for flat space, where $\mathbf{k} \cdot \boldsymbol{\gamma}=k \mu$,

$$
\mathcal{D}_{\gamma}(k)=\int_{0}^{\eta_{0}} d \eta \mathcal{V}_{\gamma} e^{-\left[k / k_{D}(\eta)\right]^{2}}
$$

is the diffusion damping factor and the visibility function $\mathcal{V}_{\gamma}=\dot{\tau} e^{-\tau}$ is the probability of last scattering within $d \eta$ of $\eta$. Its peak is near $\tau\left(z_{*}\right)=1$. The damping factor is dependent only on the background cosmology and fits across the whole range of parameter space are presented in $\mathrm{Hu} \&$ Sugiyama $(1995 \mathrm{c})$. We shall examine the meaning and implications of each of these terms below.

The baryons do not decouple from the photons precisely at last scattering. As we have seen in $\S 3.2$, the coupling strength is altered by a factor of $R, \dot{\tau}_{d}=\dot{\tau} / R$. By analogy to the photon case, we can define a drag optical depth $\tau_{d}$ and the end of the drag epoch as $\tau_{d}\left(z_{d}\right)=1$ ( $\mathrm{Hu} \&$ Sugiyama 1995c). After this point, Compton drag on the baryons can no longer prevent the gravitational infall of the baryons. Because $R\left(z_{*}\right) \sim 0.3$ for standard recombination and a big bang nucleosynthesis value for $\Omega_{b} h^{2}, z_{*}$ and $z_{d}$ approximately coincide for the standard case. However for reionized scenarios $R\left(z_{*}\right) \gg 1$ and the baryons decouple from the photons long before last scattering. It is thus no longer appropriate to consider the photons and baryons as tightly coupled at last scattering. We will develop new techniques to handle this situation in $\S 4$. From the drag depth $\tau_{d}$, a drag visibility function $\mathcal{V}_{b} \approx \dot{\tau}_{d} e^{-\tau_{d}}$ can be constructed (see Hu \& Sugiyama 1995c for the small correction due to expansion damping). The final scale for Silk damping is obtained from the visibility function and the diffusion length as in equation (15) for the photons. 

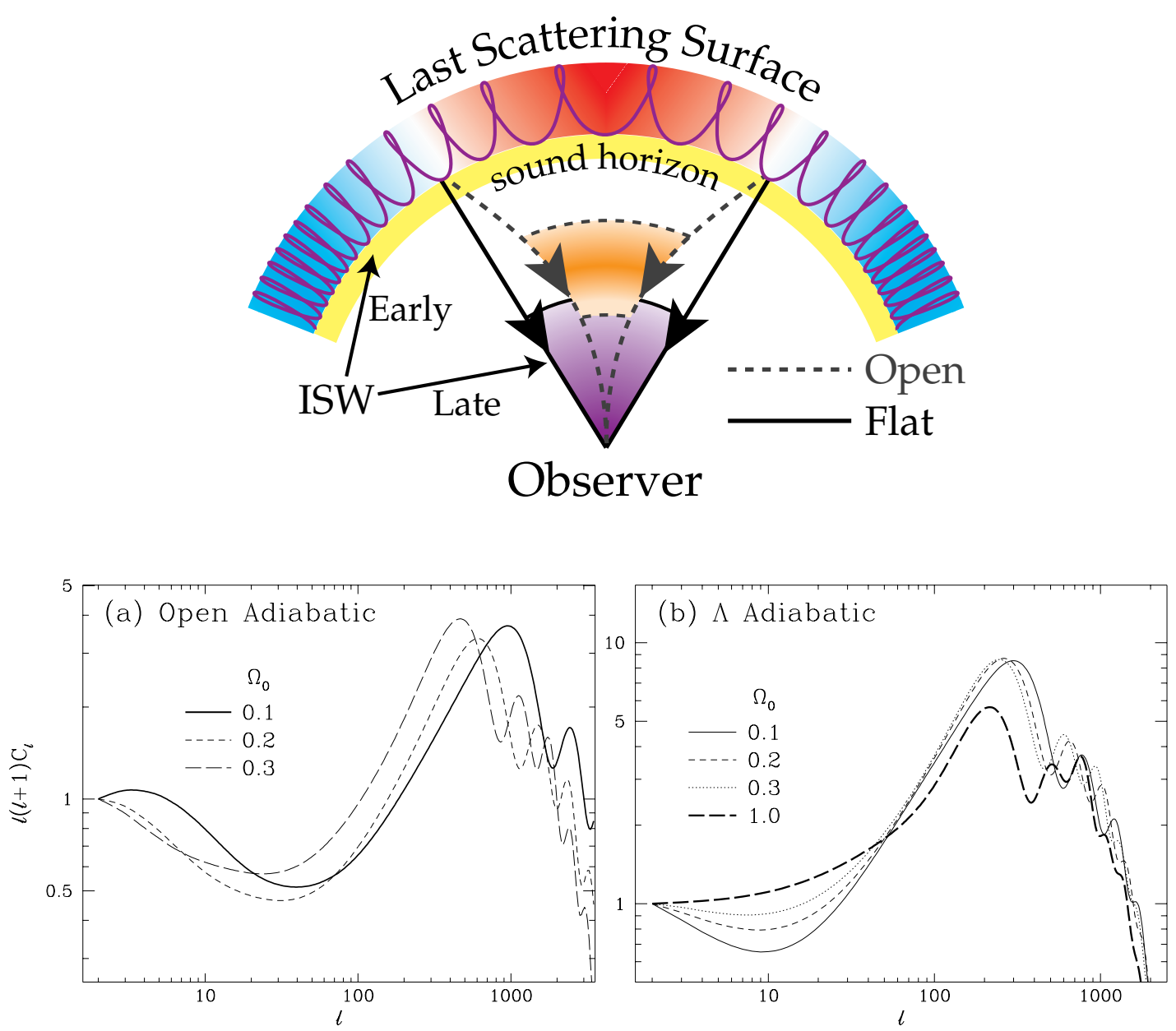

Fig. 7. Location of the peaks. The location of the peaks is determined by the angle the sound horizon subtends at last scattering. In an open universe, geodesic deviation makes this angle much smaller than in a flat universe. As this angle involves a ratio of the sound horizon to the (angular diameter) distance to last scattering, distance scale changes through $\Lambda$ and $h$ have little effect on its angle. The drop in the lowest multipoles for the open $\Omega_{0}=0.1$ is due to the lack of supercurvature scale power in the otherwise scale invariant initial conditions assumed here. Models here have $h=0.5$ and $\Omega_{b}=0.05$.

\subsection{Projection Effects and the Anisotropy Spectrum}

The presence of the $\exp (i k \mu \Delta \eta)$ term in equation (14) represents the free streaming of the photons in flat space. Photons travel at the speed of light so that the number of wavelengths traveled between last scattering and today is $k \Delta \eta$. However, the phase of the wavefront changes only in the perpendicular direction so that the observed phase change will depend on the line of sight $\phi=k \Delta \eta \mu$. Since inhomogeneities at the last scattering surface appear as anisotropies on the sky today, this can equivalently be viewed as a simple projection of the plane wave on the sphere. The anisotropy is expressed through the decomposition of the plane wave into multipole moments $\ell \sim$ 


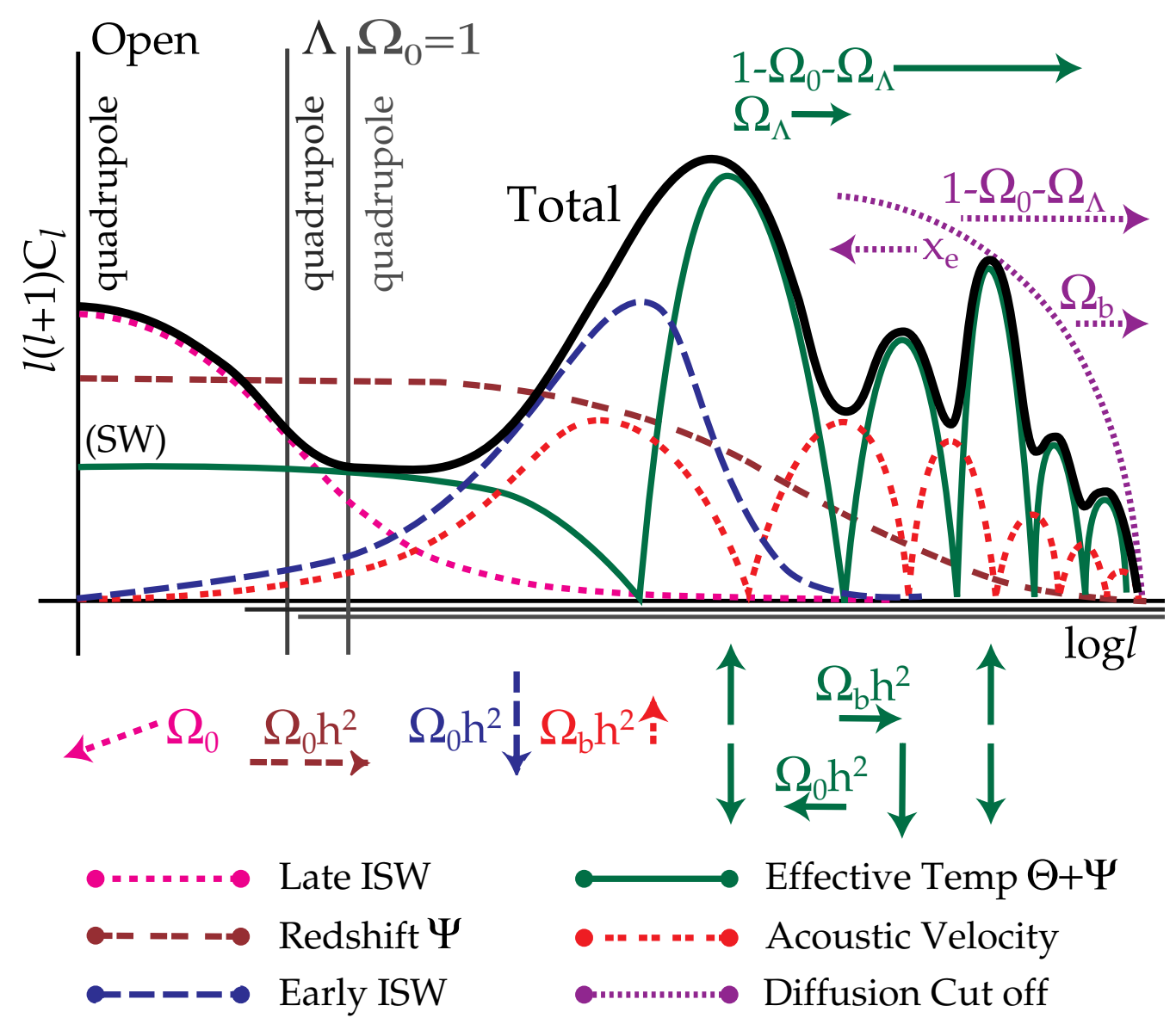

Fig. 8. Parameter sensitivity. A schematic representation based on a scale invariant adiabatic scalar model. Features in an open model are shifted to significantly smaller angles compared with $\Lambda$ and $\Omega_{0}=1$ models, represented here as a shift in the $\ell$ axis. Isocurvature models behave similarly with respect to the acoustic oscillations save that the peaks are 90 degrees out of phase. The spectrum may also be tilted by changing the initial power spectrum from the scale invariant assumption.

$\theta^{-1}$

$$
\frac{\Theta_{\ell}\left(\eta_{0}, k\right)}{2 \ell+1}=\left\{\left[\Theta_{0}+\Psi\right]\left(\eta_{*}, k\right)+\Theta_{1}\left(\eta_{*}, k\right) \frac{1}{k} \frac{d}{d \eta}\right\} \mathcal{D}_{\gamma}(k) j_{\ell}(k \Delta \eta) .
$$

Since $j_{\ell}$ peaks at $\ell \sim k \Delta \eta$, it is clear that free streaming just projects the physical scale $k \sim \lambda^{-1}$ onto an angular scale as $\theta \sim \lambda / \Delta \eta$. The total power in the $\ell$ th multipole is obtained by integration over $k$ modes, $C_{\ell}=$ $(2 / \pi) \int k^{3}\left|\Theta_{\ell}\left(\eta_{0}, k\right)\right|^{2} /(2 \ell+1)^{2} d \ln k$.

The physical content of the generalization to open universes is now obvious. Since free streaming merely represents a projection, one replaces the comoving distance $\Delta \eta$ with the comoving angular diameter distance 
$r_{\theta}=|K|^{-1 / 2} \sinh \left[|K|^{1 / 2} \Delta \eta\right]$, where the curvature $K=-H_{0}^{2}\left(1-\Omega_{0}-\Omega_{\Lambda}\right)$. For a $K>0$ closed geometry, merely replace the sinh with sin. Notice that in the closed case, the location of the first peak has oscillates with the curvature (White \& Scott 1995). Putting this together, the acoustic peaks occur at

$$
\ell_{j}=k_{j}\left|r_{\theta}\left(\eta_{*}\right)\right|=\left|\frac{r_{\theta}}{r_{s}}\right|_{\eta_{*}} \times \begin{cases}j \pi, & \text { adiabatic } \\ (j-1 / 2) \pi . & \text { isocurvature }\end{cases}
$$

Formally, this arises by replacing $j_{\ell}$ with the radial eigenfunction of an open universe (Wilson 1983). Notice that the peak locations only depend on the background cosmology once the adiabatic or isocurvature nature of the fluctuations has been established. Indeed, the ratio of the peak locations themselves can be employed to separate the two (Hu \& Sugiyama 1995b). The dominant factor in the peak locations is the curvature of the universe which can make the same physical scale subtend a much smaller angle on the sky (see Fig. 7). Here we have a classical cosmological test of the curvature: knowing the physical scale (sound horizon at last scattering) and the redshift $\left(z_{*} \approx 1000\right)$ of the acoustic peak, we measure its angular extent. The inferred angular diameter distance tells us the curvature. If the peak location is known to sufficient accuracy, we can also measure $\Omega_{b} h^{2}, \Omega_{0} h^{2}$ and $\Lambda$ through their effects on the sound horizon and angular diameter distance (see Figs. $6 \& 7$ ). Analogous information can be obtained from the location of the diffusion damping scale even if oscillations are not apparent due to random driving effects.

The heights of the peaks also contain important, if less model independent information. As we have seen, every other peak is boosted by the baryon content due to baryon drag and scales inside the horizon at equality probe $\Omega_{0} h^{2}$ or more generally the matter-radiation ratio which includes information on the neutrino content and mass. Since these two effects have relatively robust features, alternating peak heights and a strong height boost at matter-radiation equality, there is hope of obtaining information out of the heights of the peaks as well as their location for a wide class of models. As an example, we present a schematic picture for the anisotropy spectrum in Fig. 8 based on the popular scale invariant adiabatic model. Note that changing the overall dynamics from $\Omega_{0}=1$ through flat $\Lambda$ models to open models is similar to shifting the spectrum in angular space toward smaller angles. 


\section{Secondary Anisotropies and the Weak Coupling Approximation}

We have been assuming up to this point that CMB fluctuations are frozen in at a redshift $z_{*} \approx 1000$. Several processes in the foreground of recombination could alter the anisotropy spectrum. These are generally known as secondary anisotropies. Just as the tight coupling approximation assisted in the calculation and interpretation of primary anisotropies, the weak coupling approximation helps in understanding secondary anisotropies ( $\mathrm{Hu} \&$ White 1995).

In abstract form, anisotropy generation is governed by internal sources from photon fluctuations at last scattering $S_{\text {prim }}$ and sources external to the photon system in the foreground of recombination $S_{\text {sec }}$ by

$$
\frac{\Theta_{\ell}\left(\eta_{0}, k\right)}{2 \ell+1}=\int_{0}^{\eta_{0}}\left[S_{p r i m}+S_{s e c}\right] j_{\ell}(k \Delta \eta) d \eta
$$

in flat space with an appropriate generalization of the radial eigenfunction for an open geometry (Wilson 1983). Equation (17) just states that we observe the projection of the source at $\eta$ on a shell at a distance $\Delta \eta=\eta_{0}-\eta$. Whether the sources or the phase of the wave varies more rapidly distinguishes the tight from the weak coupling regime. For primary anisotropies, the source is localized over a short range of time around recombination. Since $\dot{\tau} / k \gg 1, j_{\ell}$ can be taken out of the integral (17) leading to the simple projection of equation (16).

Secondary anisotropies do not necessarily possess this property. Most cosmological effects aside from decoupling take on the order of an expansion time at the relevant epoch to be completed. In these cases, $S_{\text {sec }}$ may be taken to be slowly varying at small scales and removed from the integral in (17). Since

$$
\int_{0}^{\eta_{0}} j_{\ell}(k \Delta \eta) d \eta \approx \frac{\sqrt{\pi}}{2 k} \frac{\Gamma\left[\frac{1}{2}(\ell+1)\right]}{\Gamma\left[\frac{1}{2}(\ell+2)\right]} \approx \sqrt{\frac{\pi}{2 \ell}} \frac{1}{k}
$$

in the weak coupling limit where $\dot{S}_{\text {sec }} /\left(k S_{\text {sec }}\right) \ll 1$, anisotropies fall with $\ell$ more rapidly than a simple projection of the source would imply. This just reflects the fact that it contributes across many wavelengths of the fluctuation allowing contributions from crests and troughs to cancel. We will now discuss the main sources of secondary anisotropies $S_{\text {sec }}$. 


\subsection{Gravitational Effects}

Even in the absence of reionization, secondary anisotropies can be generated by gravitational redshift effects between recombination and today. The differential redshift from $\dot{\Psi}$ and dilation from $\dot{\Phi}$ discussed above must be integrated along the trajectory of the photons $S_{s e c}=\dot{\Psi}-\dot{\Phi}$. Tensor fluctuations can also give rise to anisotropies through this mechanism. We thus call the combination the integrated Sachs-Wolfe (ISW) effect. Notice that these effects only occur if the metric fluctuation is time varying. We can separate this general mechanism for anisotropy formation into categories based on the reason for the time evolution. There are four possibilities to consider: time evolution due to the radiation content (early ISW effect), due to the expansion (late ISW effect), due to non-linear evolution (Rees-Sciama effect) and any more exotic sources such as gravity waves or defects (sourced ISW). Additionally, beyond linear theory gravitational lensing may affect the CMB. Since this merely shuffles power in anisotropies around scales at the arcminute level for a CDM type model, we will not further consider the effect and refer the interested reader to Seljak (1995a) and references therein.

\subsubsection{Early ISW Effect}

The early ISW effect is the direct analogue of the acoustic driving effect of $\S 3.1 .4$ except that the photons are in the free streaming rather than the tight coupling regime. For adiabatic conditions, the potential decays after horizon crossing in the radiation dominated limit. For isocurvature conditions, it grows outside the horizon and then decays as in the adiabatic case. In general, this effect will smoothly match onto the acoustic peaks due to similarities in the cause of their generation. Due to the later time of generation, this effect influences larger scales than the acoustic peak but is cut off above the equality scale. Furthermore, since it arises from a distance closer to ourselves, the same physical scale subtends a larger angle on the sky. Together these considerations imply that the early ISW effect fills in the anisotropy on scales just larger than the first acoustic peak (see Fig. 8). It serves to broaden the rise and shifts the location of the first peak in the spectrum to larger scales. Unfortunately, this effect satisfies neither the tight nor the weak coupling approximation since the decay time $\sim \eta$ and the wavelength $\sim k^{-1}$ are by definition comparable for this horizon crossing effect. 


\subsubsection{Late ISW Effect}

If the universe has a non-vanishing curvature or cosmological constant, eventually these will dominate the expansion rate. Under the rapid expansion, density fluctuation $\delta \rho_{T}$ decays taking the potential with it. The decay takes on the order of an expansion time at the end of matter domination independent of the wavelength. Since the photons free stream, they travel across many wavelengths of the perturbation on scales smaller than the horizon. If the potential decays while the photon is in an underdense region, it will suffer an effective redshift rather than a blueshift. Contributions from overdense and underdense regions will cancel and damp the ISW effect on small scales. This is the hallmark of the weak coupling regime and is mathematically expressed through cancellation in the integral (18).

For a fixed $\Omega_{0}$, the decay epoch occurs much later in flat $\Omega_{\Lambda}+\Omega_{0}=1$ models than open ones. Thus $\Lambda$ models will suffer cancellation of late ISW contributions at a much larger scale than open models. In fact, for reasonable $\Omega_{0} \gtrsim 0.1$ decay the decay has already started at the quadrupole (see Fig. 7,8; Kofman \& Starobinskii 1985; Hu \& White 1995). In summary, the epoch that the universe exits the matter dominated phase is imprinted on the CMB by the late ISW effect.

\subsubsection{Rees-Sciama Effect}

Even for an $\Omega_{0}=1 \mathrm{CDM}$ dominated universe, potentials only remain constant in linear perturbation theory. The second order contribution has been shown to be negligibly small (Martinez-Gonzalez, Sanz \& Silk 1992). The effect in the non-linear regime has been estimated with N-body simulations through power spectrum techniques (Seljak 1995) and ray tracing techniques (Tuluie, Laguna \& Anninos 1995). The general conclusion is that this effect does not become comparable to the primary signal until well into the diffusion damping tail. In the absence of reionization, it should therefore present no problem for the extraction of main features such as the curvature and the baryon content from the acoustic peaks. It may however complicate the extraction of cosmological parameters from more subtle effects, for example the neutrino mass.

\subsubsection{Sourced ISW Effect}

This catch-all category contains all other gravitational redshift effects on the CMB. Tensor perturbations in the metric, i.e. gravity waves, give rise to dilation effects as the photons free stream. Due to the nature of the metric distortion, they leave a quadrupole signature in the CMB which thereafter is projected onto higher multipoles. Combined with cancellation effects, this implies that the tensor spectrum thus typically exhibits a sharp drop in power from the quadrupole. Since contributions only arise between 


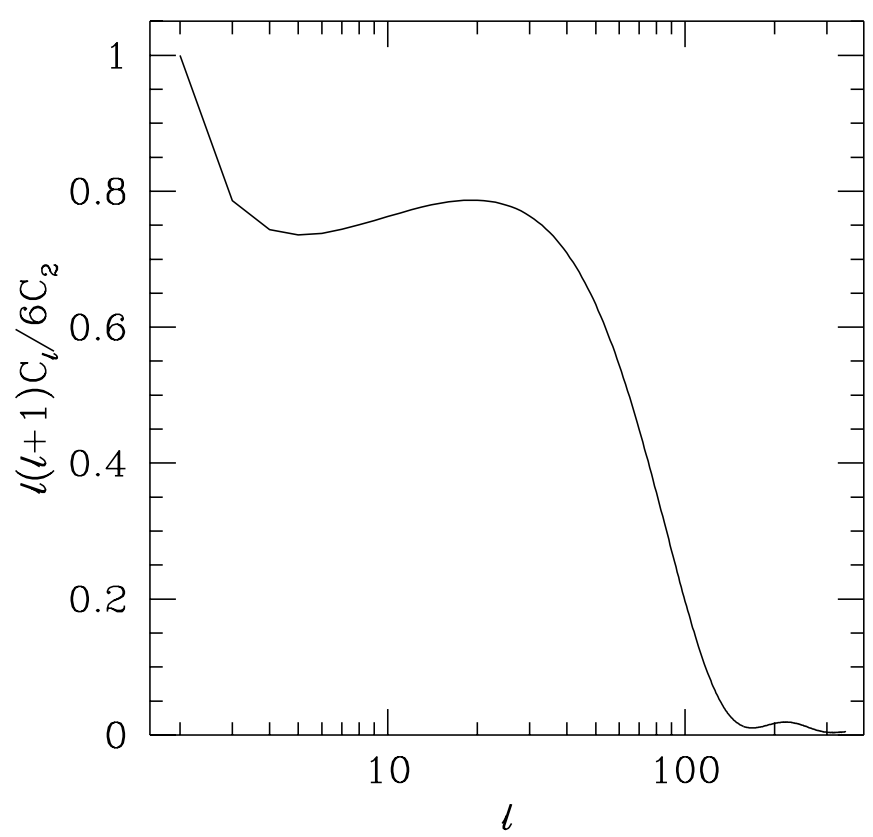

Fig. 9. Gravitational Wave ISW Effect. The metric distortion caused by a gravitational wave induces a redshift/dilation effect on CMB photons. The spectrum is cut off below the angle subtended by the horizon at last scattering since gravity waves only affect free streaming photons significantly. Due to the signature of the metric distortion, it originates as a source of the quadrupole anisotropy.

last scattering and the present, there is also a cut off at the angle the horizon subtends at last scattering. Below this scale, the gravity waves have already redshifted away by last scattering. Detailed calculations of the spectrum were first carried out by Crittenden et al. (1994) and the spectrum shown in Fig. 9 is from $\mathrm{Hu}$ et al. (1995). For inflationary models, there exists a consistency relationship between the amplitudes of the scalar and tensor modes and the slope of the power spectrum (see e.g. Steinhardt 1995). There is hope that detailed measurements of the CMB spectrum can thus provide a strong test of the inflationary scenario (see Lindsey et al. 1995 and references therein).

Cosmological defects may also act as an external source of gravitational potential perturbations. Pen, Spergel \& Turok (1993) find that in such models a scale invariant form for the anisotropy results from this effect and can mimic the inflationary prediction. As in the case of the late ISW effect, the magnitude drops from $2 \Psi$ to zero as the characteristic time scale for the change in $\Psi$ becomes longer than the light travel time across a wavelength. For defect models Pen, Spergel \& Turok (1993) estimate the amplitude as approximately $\frac{4}{3} \Psi$. Since this effect occurs around horizon crossing for a given mode due to the isocurvature conditions, the largest spatial modes 


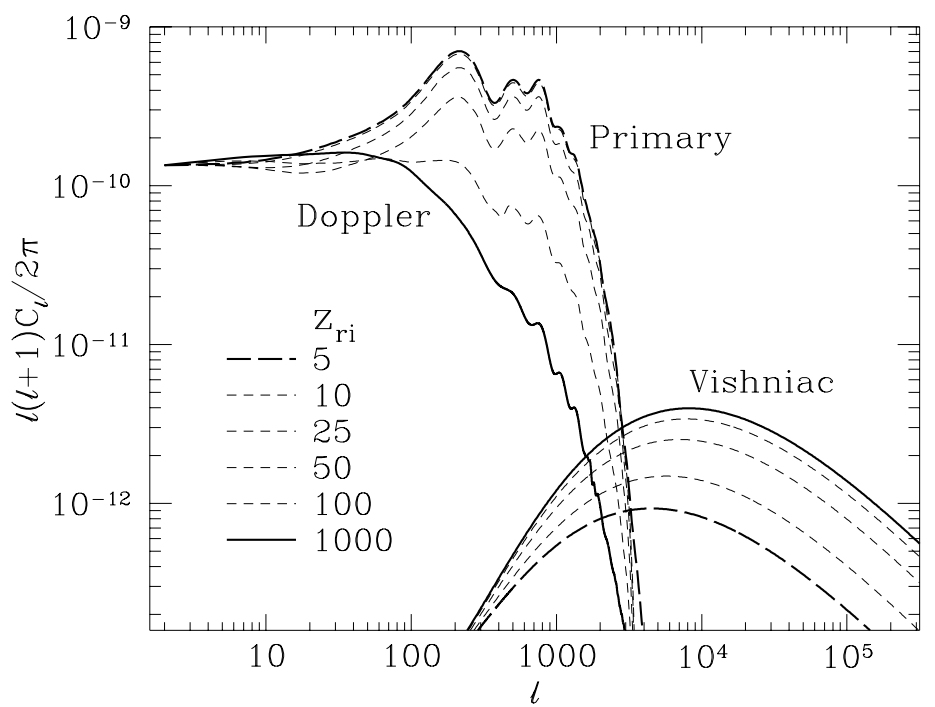

Fig. 10. Reionization damping, Doppler, and Vishniac effects (scale invariant adiabatic $\Omega_{0}=1, \Omega_{b}=0.05, h=0.5$, COBE normalized model). As the ionization level between recombination and the present increases, primary anisotropies are reduced by diffusion and rescattering. For sufficiently high ionization, here $z_{i} \gtrsim 100$, the Doppler effect on the new last scattering surface can regenerate some fluctuations at intermediate scales. The Vishniac effect dominates at very small angular scales. Due to its second order nature, it contributes even if the ionization redshift is low. The $z_{i}=5,10$ curves are indistinguishable for the primary anisotropies as are the $z_{i}=100,1000$ for the Vishniac effect.

have not been enhanced and can lead to a drop in power at the lowest multipoles.

\subsection{Scattering Effects}

An early round of structure formation may be able to reionize all or part of the universe at high redshift. If this occurs at

$$
z \gtrsim z_{*} \approx 10^{2}\left(\Omega_{0} h^{2} / 0.25\right)^{1 / 3}\left(x_{e} \Omega_{b} h^{2} / 0.0125\right)^{-2 / 3}
$$

where $\tau\left(z_{*}\right)=1$ then CMB anisotropies will be drastically influenced by scattering. The model-independent information stored in the primary anisotropy spectrum will be greatly reduced. It is replaced by more detailed information and clues about the evolution of structure in the universe. 


\subsubsection{Reionization Damping}

The most dramatic effect of reionization is the increase in the photon diffusion length. Since last scattering is delayed until the Compton mean free path approaches the horizon (or equivalently when the scattering rate $\dot{\tau}$ drops below the expansion rate $\dot{a} / a)$, the diffusion length at last scattering is the horizon scale $\lambda_{D} \approx \sqrt{\eta / \dot{\tau}} \approx \sqrt{\eta a / \dot{a}} \approx \eta$. Intrinsic photon fluctuations such as the acoustic oscillations will be damped by diffusion below the horizon scale. Moreover, since acoustic oscillations appear only below the sound horizon no oscillations will be apparent in the reionized spectrum.

If reionization is not sufficiently early, some trace of the acoustic oscillations may remain in the spectrum. Recall that diffusion damping works since rescattering of photons arriving from directions with different intrinsic temperatures varies destroys the anisotropy. Fluctuations are only retained in the unscattered fraction $e^{-\tau}$. If the total optical depth between recombination and the present is $\tau \lesssim 1$, primary and secondary scattering anisotropies may be present in the spectrum (see Fig. 10). In this case, the information contained in the CMB would truly be enormous but also difficult to extract.

\subsubsection{Cancelled Doppler Effect}

After the Compton drag epoch $\frac{3}{5} z_{d} \approx 165\left(\Omega_{0} h^{2}\right)^{1 / 5} x_{e}^{-2 / 5}$ where $\tau_{d}\left(z_{d}\right)=1$ (Hu \& Sugiyama 1995c), baryonic gravitational instability can no longer be prevented by the photons. Collapse of the baryon density fluctuations implies that baryon peculiar velocities will create a Doppler effect in the CMB. However the weak coupling approximation tells us that these too will be damped as the photon traverses many crests and troughs of the perturbation at last scattering. In fact, cancellation for the Doppler effect is particularly severe. If the perturbation wavevector is perpendicular to the line of sight $\mathbf{k} \perp \gamma$, cancellation is avoided. However, flows are irrotational in linear theory so $\mathbf{v}_{b} \| \mathbf{k}$. Since the velocity is then perpendicular to the line of sight, no Doppler effect arises $\gamma \cdot \mathbf{v}_{b}=v_{b} \gamma \cdot \hat{\mathbf{k}}=0$ (see Fig. 11). Fluctuations only survive if there are variations in the velocity or optical depth through last scattering. Thus the Doppler source is related to the derivative of these quantities as $S_{\text {sec }}=e^{-\tau}\left[\dot{V}_{b} \dot{\tau}+V_{b} \ddot{\tau}\right] / k$ at small scales where recall $\mathbf{v}_{b}(\mathbf{x})=-i V_{b} e^{i \mathbf{k} \cdot \mathbf{x}} \hat{\mathbf{k}}$. This leads to a suppression by a factor of approximately $\left(k \eta_{*}\right)^{-1}$ (Kaiser 1984). Cancellation under the weak coupling approximation yields a further suppression via equation (18). In general, later last scattering implies a greater $k \eta_{*}$, a larger cancellation scale, and hence smaller Doppler fluctuations. Nevertheless, in Fig. 10 the slight upturn at intermediate scales for the high ionization case is due to this effect. 

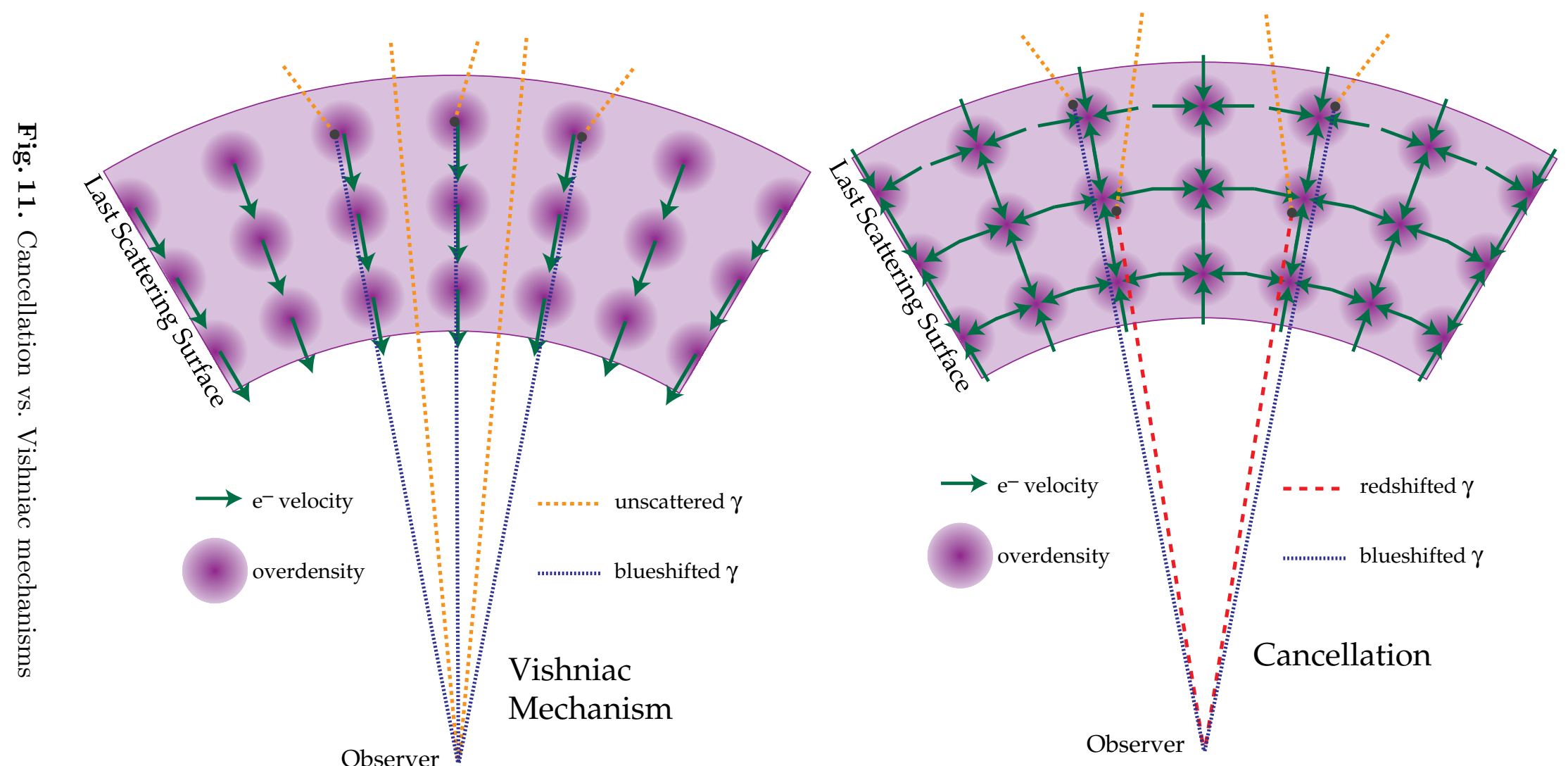

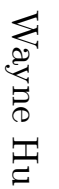
Observer

Observer 


\subsubsection{Vishniac Effect}

Since the first order Doppler fluctuation is severely suppressed due to "coincidental" geometrical reasons other effects may dominate the anisotropy at small scales. Variations in the optical depth due to density fluctuations across the last scattering surface may alow the Doppler effect to escape severe cancellation. The increased probability of scattering causes a preferential generation of Doppler fluctuations in overdense regions. The ensuing anisotropy is not as severely damped as the first order contribution. This is because the velocity field can be parallel to the line of sight while the density variation is perpendicular to the line of sight (see Fig. 11). This effect can also be calculated under the weak coupling approximation. It is slightly more complicated than linear effects since it entails mode coupling between the density and velocity fields. An explicit expression is given in $\mathrm{Hu}$ \& White (1995). For a CDM model, we find that Vishniac contributions do indeed exceed linear contributions at sufficiently small scales. Furthermore, since second order effects are strongly peaked to late times, even minimally ionized scenarios carry a significant fraction of the maximum signal (see Fig. 10).

\subsubsection{Cluster Sunyaev-Zeldovich Effect}

Clusters provide a non-linear analogue of the Vishniac effect. Here the hot cluster provides the variation in the optical depth which causes preferential scattering. The Doppler effect due to the peculiar velocity of the cluster yields an anisotropy known as the kinematic Sunyaev-Zel'dovich (SZ) effect (Sunyaev \& Zel'dovich 1972). For an individual cluster the temperature fluctuation is of order $\tau_{c} v_{c}$, where the optical depth through a cluster is of order $\tau_{c} \approx 0.1-0.01$ and the peculiar velocity $v_{c} \approx$ few $\times 10^{-3}$. This provides an interesting way of measuring the peculiar velocity of a cluster without introducing the problems associated with determining the distance scale (see e.g. Sunyaev \& Zel'dovich 1980, Haehnelt \& Tegmark 1995). The average effect is much smaller however and probably does never dominates the anisotropy spectrum. Persi et al. (1995) estimate the effect in a CDM model from hydrodynamic simulations and find that this non-linear Doppler effect is small in comparison to the second order Doppler (Vishniac) effect

Compton scattering off hot electrons also produces spectral distortions which look like anisotropies to any experiment confined to low frequency measurements. As discussed in $\S 2.1$, Compton scattering exchanges energy between the electrons and photons to order $T_{e} / m_{e}$. This upscatters photons from the Rayleigh-Jeans to the Wien regime and leads to a temperature distortion of $(\Delta T / T)_{R J}=-2 y \approx-2 \tau_{c} T_{e} / m_{e} \approx 10^{-5}-10^{-3}$ for an individual cluster. Again, the rms fluctuations would be much smaller. Much effort has been expended to estimate the fluctuations caused by this thermal SZ effect with varying results (Barbosa et al. 1995; Makino \& Suto 1993; Cole 
\& Kaiser 1988). Recently, empirical modeling of clusters has predicted that the anisotropy at arcminutes should be on the order of $(\Delta T / T)_{R J} \lesssim 10^{-7}$ (Ceballos \& Barcons 1994). Moreover the signal is in large part due to bright and easily identifiable clusters. If such known clusters are removed from the sample, the anisotropy drops to an entirely negligible level.

\subsubsection{Inhomogeneous Reionization}

Finally, let us mention another variant of the Vishniac-Sunyaev-Zel'dovich mechanism. The optical depth could vary due to inhomogeneities in the ionization fraction $x_{e}$ near last scattering. Note that inhomogeneities well before optical depth unity have little effect on the CMB due to the cut off from the visibility function. Details of this effect will of course depend on the exact model for reionization, i.e. the extent of the inhomogeneities. Small inhomogeneities may be expected to behave as the Vishniac effect; large inhomogeneities like the kinetic SZ effect.

\section{Discussion}

It should now be clear that a great wealth of information about cosmology and the model for structure formation lies waiting to be observed in the CMB anisotropy spectrum. The location of the first acoustic peak provides a robust classical test for curvature in the universe if the fluctuations are known to be either adiabatic or isocurvature. The only caveat here is that if the universe suffered early reionization the acoustic effect may be so suppressed that it becomes unobservable. In this case, the CMB will place a lower limit on the epoch of reionization. It is very likely that the curvature and/or thermal history of the universe will be measured in the near future.

Barring early reionization, once we obtain precise measurements of the first peak and beyond, we should be able to extract much more information. The relative locations of the higher peaks can cleanly separate adiabatic and isocurvature models independently of the curvature and thermal history. A precise measurement of the location of the peaks can supply information on $\Lambda$ and $\Omega_{0} h^{2}$. The heights of the peaks yield even more information if some minimal assumptions are made for the theory of structure formation. Indeed, requiring consistency with large scale structure measurements should eventually fix the model quite precisely. The relative heights of the peaks give a robust probe of the baryon content $\Omega_{b} h^{2}$. In any given model, the absolute heights yield a constraint on the matter content of the universe $\Omega_{0} h^{2}$ and perhaps the number, temperature and mass of the neutrinos. Combining these pieces of information we can infer the Hubble constant.

It would seem that all the fundamental cosmological parameters are encoded in the CMB anisotropy spectrum. Yet, even discounting the possibility of early reionization, how likely is it that we will precisely measure 
them with the next generation of experiments? The results of Jungman et al. (1995) suggest that at least the curvature can be measured to better than $5 \%$ accuracy with a full sky map to half a degree resolution. Precisely how much information can be extracted will depend in the end on how severe foreground contamination from synchotron radiation, free-free emission, interstellar cold dust, and radio point sources will be. Many of the effects described here will require $\Delta T / T$ to $10^{-6}$ accuracy to measure definitively. With sufficient frequency coverage, there is hope of distinguishing the background from the foreground signal to employ at least the clean patches of the sky for cosmology.

Acknowledgments: I would like to thank E. Bunn, J. Silk, D. Scott, N. Sugiyama, M. White as the ideas and results presented here arise from our many collaborations. In particular, numerical results presented here are from the code of N. Sugiyama unless otherwise stated. M. Tegmark provided useful comments on a draft of these notes. I would also like to thank the organizers of this school E. Martinez-Gonzalez and J.L Sanz for an enjoyable and productive meeting.

\section{References}

Albrecht, A., Coulson, D., Ferreira, P. \& Magueijo, J. (1995): astroph-9505030

Barbosa, D., Bartlett, J.G., Blanchard, A. \& Oukbir, J. (1995): astroph-9511084 Bardeen, J.M. (1980): Phys. Rev. D 221882

Bond, J.R. (1995): "Theory and Observations of the CBR", in Cosmology and Large Scale Structure, ed. by Schaeffer (Elsevier, Netherlands)

Bond, J.R. \& Efstathiou, G. (1984): ApJ Lett. 285 L45

Bond, J.R. et al. (1994): Phys. Rev. Lett. 7213

Cole, S. \& Kaiser, N. (1988): MNRAS 233637

Crittenden, R. et al. (1994): Phys. Rev. Lett. 71324

Crittenden, R. \& Turok, N. (1995): astroph-9505120

de Laix, A.A. \& Scherrer, R.J. (1995): astroph-9509075

Dodelson, S., Gates, E. \& Stebbins, A. (1995): astroph-9509147

Dodelson, S. \& Jubas, J. (1995): ApJ 439503

Doroshkevich, A.G., Zel'dovich, Ya.B. \& Sunyaev, R.A. (1978): Sov. Astron. 22 523

Durrer, R., Gangui, A. \& Sakellariadou (1995): astroph-9507035

Haehnelt, M.G. \& Tegmark, M. (1995): astroph-9507077

$\mathrm{Hu}$, W. (1995): astroph-9508126

Hu, W., Scott, D. \& Silk, J. (1994): Phys. Rev. D. 49648

Hu, W., Scott, D., Sugiyama, N. \& White, M. (1995): Phys. Rev. D (in press, astroph-9505043)

Hu, W. \& Sugiyama, N. (1995a): ApJ 436456

Hu, W. \& Sugiyama, N. (1995b): Phys. Rev. D 512599

Hu, W. \& Sugiyama, N. (1995c): astroph-9510117 
Hu, W. \& White, M. (1995): A\&A (in press, astroph-9507060)

Jungman, G., Kamionkowski, M., Kosowsky, A. \& Spergel, D.N. (1995): astroph9507080

Kaiser, N. (1983): MNRAS 2021169

Kaiser, N. (1984): ApJ 282374

Kodama, H. \& Sasaki, M. (1984): Prog. Theor. Phys. Supp. 781

Kofman, L.A. \& Starobinskii, A.A. (1985): Sov. Astron. Lett. 9643 (1985)

Kosowsky, A. (1995): astroph-9501045

Lidsey, J.E. et al. (1995): astroph-9508078

Ma, C.-P. \& Bertschinger, E. (1995): astroph-9506072

Magueijo, J., Albrecht, A., Coulson, D. \& Ferreira, P. (1995): astroph-9511042

Makino, N. \& Suto, Y. (1993): ApJ 4051

Martinez-Gonzalez, E., Sanz, J.L. \& Silk, J. (1992): Phys. Rev. D 464193

Mukhanov, V.F., Feldman, H.A., \& Brandenberger, R.H. (1992): Phys. Rep. 215 203

Ostriker, J.P. \& Vishniac, E.T. (1986): ApJ 30651

Peebles, P.J.E. \& Yu, J.T. (1970): ApJ 162815

Pen, U.-L., Spergel, D.N. \& Turok, N. (1994): Phys. Rev. D 49692

Persi, F.M., Spergel, D.N., Cen, R. \& Ostriker, J.P. (1995): ApJ 4421

Press, W. \& Vishniac, E.T. (1980): ApJ 2391

Rees, M.J. \& Sciama, D.N. (1968): Nature 519611

Sachs, R.K. \& Wolfe, A.M. (1967): ApJ 14773

Scott, D., Silk, J. \& White, M. (1995): Science 268829

Seljak, U. (1994): ApJ Lett. 419 L47

Seljak, U. (1995a): astroph-9506048

Seljak, U. (1995b): astroph-9505109

Silk, J. (1968): ApJ Lett. 151459

Smoot, G. et al. (1992): ApJ Lett. 396 L1

Steinhardt, P.J. (1995): astroph-9502024

Sugiyama, N. \& Gouda, N. (1992): Prog. Theor. Phys. 88803

Sunyaev, R.A. \& Zel'dovich, Ya. B. (1972): Comm. Astrophys. Space Phys. 473

Sunyaev, R.A. \& Zel'dovich, Ya. B. (1980): MNRAS 190413

Tuluie, R. \& Laguna, P. (1995): ApJ Lett. 445 L73

Vishniac, E.T. (1987): ApJ 322597

Vittorio, N. \& Silk, J. (1984): ApJ Lett. 285 L39

Weinberg, S. (1972): Gravitation and Cosmology (Wiley, New York)

White, M., \& Scott, D. (1995): astroph-9508157

White, M., Scott, D. \& Silk, J. (1994): ARA\&A 32319 (1994)

Wilson, M.L. (1983): ApJ 2732

Wilson, M.L. \& Silk, J. (1981): ApJ 24314 